\title{
The Ecology of Gravimagnetic Rotator in the past 30 years
}

\author{
Vladimir Lipunov ${ }^{1}$ \\ Lomonosov MSU - Lomonosov Moscow State University, Physics Department, SAI Universitetsky prt.13, \\ Moscow, 119234 Russia \\ E-mail: lipunovesai.msu.ru
}

Daniil Vlasenko

Lomonosov Moscow State University, Physics Department, SAI

Universitetsky prospect, 13, Moscow, Russia 119234

E-mail: Iipunovesai.msu.ru

\section{Valeria Grinshpun}

Lomonosov Moscow State University, Physics Department, SAI

Universitetsky prospect, 13, Moscow, Russia 119234

E-mail: Iipunovesai.msu.ru

The compact sources discovered in recent decades in the Milky Way and neighboring galaxies are considered within the framework of the gravimagnetic rotator paradigm (GMR). GMR is characterized by mass, magnetic field and angular momentum. The rotator interacts with the environment (plasma) through the electromagnetic and gravitational fields. This rotator is a useful theoretical model for neutron stars, white dwarfs, magnetic stars, and spinars (arising immediately after the start of the collapse of double neutron stars or fast-rotating nuclei of massive stars, etc.). The ecology of rotators includes this classification, concerning their interaction with the environment, the definition of evolutionary laws and their energy interpretation. A single universal diagram of known and new types of neutron stars and white dwarfs discovered in recent years (such as ultra-bright X-ray pulsars and radio pulsars of white dwarfs) is presented, in which these objects acquired their physically transparent place in accordance with the laws of electromagnetic and gravitational interaction.

Accretion Processes in Cosmic Sources - II - APCS2018

3-8 September 2018

Saint Petersburg, Russian Federation

1

Speaker 


\section{Introduction}

The first to consider the idea that the model of a magnetic rotator can explain the energy sources of compact astrophysical objects appear to be Nikolai Semenovich Kardashev in 1964 [1] and Franco Pacini in 1967 [2]. This idea became especially popular immediately after the discovery of radio pulsars in 1968 [3] and their interpretation as magnetized neutron stars with dipole magnetic fields by Gold in 1968 [4]. On the other hand, Zeldovich and Novikov in 1966 [5] and Shklovsky in 1967 [6] paid special attention to the accretion mechanism (first proposed by Zeldovich [7] and Salpeter[8]) and to the powerful gravitational field of magnetized neutron stars. In fact, even before the discovery of radio- (in 1968) and x-ray (by Giacconi et al. in 1972 [9]) pulsars, Bisnovaty-Kogan and Friedman in 1969 [11], Zeldovich and Shakura in 1969[12], and Shvartsman in 1970 [13] -- young graduate students of Yakov B. Zeldovich and his co-authors Amnuel and Guseinov in 1967 [10] -- actually predicted the pulsar-type phenomenon in neutron stars.

The next 10 years were marked by the discovery of dozens and hundreds of objects that sometimes have very special astrophysical properties, but are also associated with compact stars like neutron stars and white dwarfs -- radio pulsars, X-ray pulsars, X-ray bursters, cataclysmic variable stars, polars, intermediate polars, millisecond radio pulsars, X-ray Novae, and SS433type sources.

So, it became necessary to have a unified approach to such a variety of objects.

As a result, a generalized model of a gravimagnetic rotator (GMR) was introduced by Lipunov in 1982 and in 1987 [14,15,16]. This generalized model was able to unite a whole variety of compact sources with different energy release mechanisms, and link them within the framework of rotational evolution paradigm.

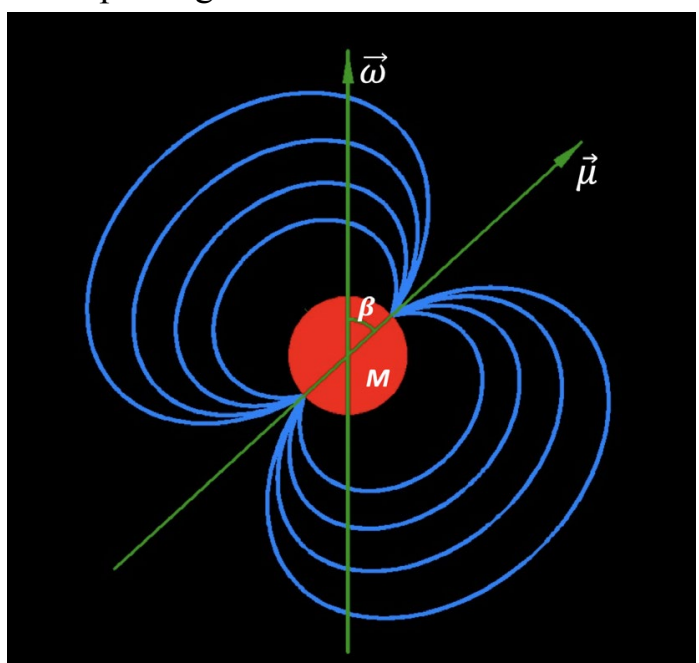

Figure 1: Gravimagnetic rotator. $\mathrm{M}, \omega, \mu$ are the mass, rotation frequency and magnetic dipole moment.

\section{The Classification of GMR}

It is clear that obtaining a solution for (gravitational and electromagnetic) interaction of such an object with the surrounding plasma capable of accretion represents the most challenging magnetohydrodynamic three-dimensional hydrodynamic problem in the gravitational field of 
the rotator. As a zero approximation, Lipunov [15] proposed in 1987 a qualitative approach to assign the main modes of interaction based on the relationship between the proncipal characteristic radius of interaction between the rotator and the environment.

These are the four characteristic radii responsible for the gravitational and electromagnetic parts:

$\boldsymbol{R}_{\text {stop }}$, the stop radius; $\boldsymbol{R}_{\boldsymbol{c}}=\left(\boldsymbol{G M} / \boldsymbol{\omega}^{2}\right)^{1 / 3}$, the corotation radius; $\boldsymbol{R}_{l}=\boldsymbol{c} / \boldsymbol{\omega}$, the light cylinder radius, and $\boldsymbol{R}_{G}=2 \boldsymbol{G M} / \boldsymbol{v}_{\infty}{ }^{2}$, the gravitational capture radius (the Bondi-Hoyle radius). In binary systems, an additional characteristic dimension $\boldsymbol{a}$ - the distance between the components -appears. In these formulas, $\boldsymbol{v}_{\infty}$ is the velocity of the rotator relative to the environment. For simplicity, we believe that the motion of the rotator is supersonic (as is usually the case under astrophysical conditions).

The stop radius is the characteristic distance at which the pressure of the electromagnetic forces preventing the accretion equals that of the forces of gravity.

$$
R_{\text {stop }}=\left\{\begin{array}{c}
R_{\text {sh }}-\text { Shwartzman radius } R_{\text {stop }}>R_{l} \\
R_{A}-\text { Alfven radius if } R_{\text {stop }}<R_{l}
\end{array}\right.
$$

In the classification, we used another parameter, namely the accretion rate. The fact is that the astrophysical properties of binary systems vary significantly if the rate of the captured substance exceeds the critical limit $\ddot{M}_{c r}$ corresponding to Eddington limit [14],[16]:

$$
\dot{M}_{c r} G M / R_{\text {stop }}=L_{E d d}=4 \pi G M c / k_{T}
$$

The maximal luminosity of the neutron star Accretor is the:

$$
L_{\max }=\dot{M}_{c r} G M / R_{x} \approx 0.1 \dot{M}_{c r} c^{2} \approx 1.310^{40} R_{8}[\mathrm{erg} / \mathrm{s}]
$$

Where $\boldsymbol{R}_{x}=10^{6} \mathbf{c m}$ is the neutron star radius, $\boldsymbol{R}_{8}=\boldsymbol{R}_{\text {stop }} / 10^{8} \mathrm{~cm}$ is the stop radius, and $\boldsymbol{k}_{T}$, the Thompson cross section for photon scattering on free electrons calculated per unit mass.

$$
\dot{M}_{c r}=4 \pi c R_{\text {stop }} / k_{T} \approx 10^{-6} R_{8} M_{\Theta} / y r
$$

Supercritical accretion rates occur in massive binary systems when a normal star fills its Roche lobe. However, this accretion mode is always implemented together with the formation of an accretion disk.

Moreover, if $\dot{\boldsymbol{M}}>\dot{\boldsymbol{M}}_{\boldsymbol{c r}}$, the radiation pressure begins to exceed gravitational pressure and excess matter is displaced from the disk surface in the form of a sort of stellar wind [17],[18].Thus, a limited set of possible modes of interaction of the gravimagnetic rotator with the accreted plasma occurs. Let us emphasize one very significant, but often misunderstood circumstance. The energy release in the case of the latter condition of the definition of the super-critical regime is determined at the stop radius, and not at the surface of the accreting star. Because of the large radii of white dwarfs and the fact that they are mainly observed in lowmass binary systems, we do not consider supercritical regimes for these objects as unlikely (see the white dwarfs section below). 
a)

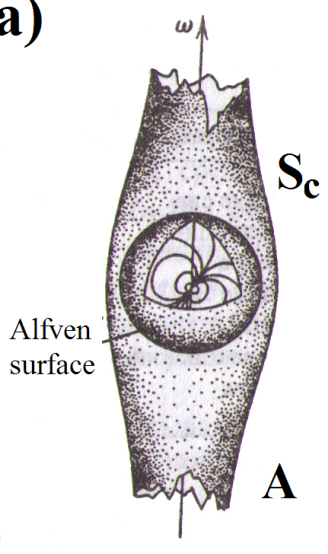

b)

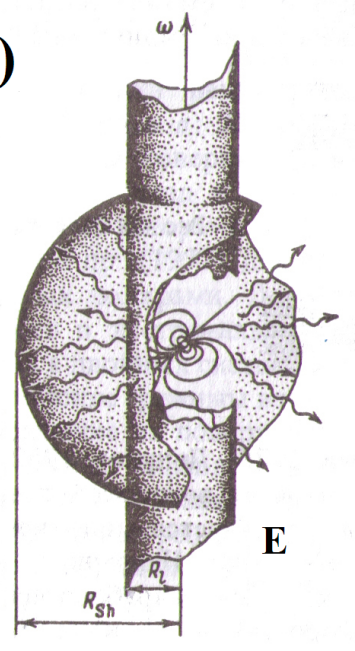

e)

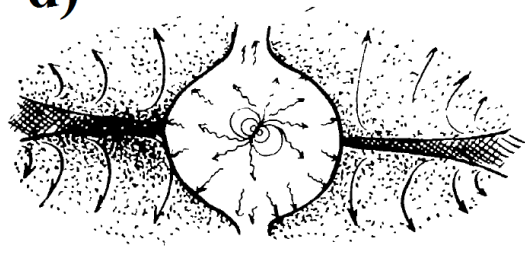

SE

g)

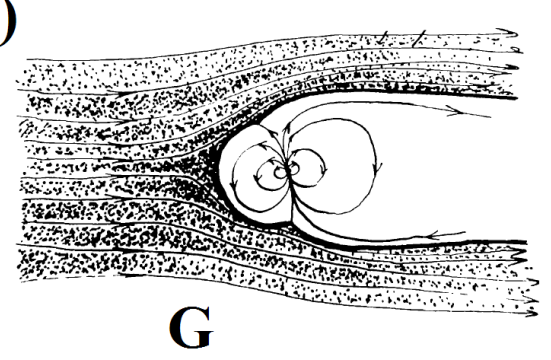

h)
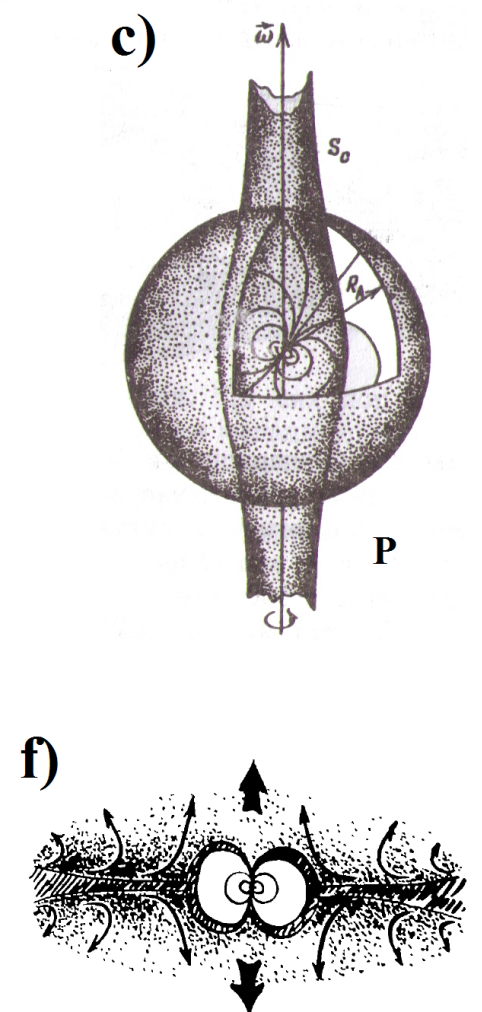

SA

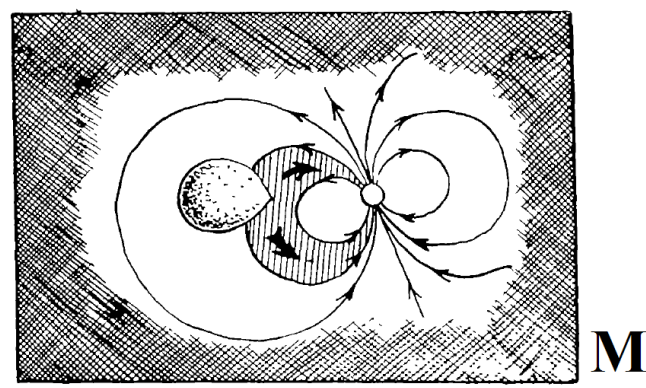

Figure 2: A qualitative picture of the interaction of a magnetic rotator with the surrounding environment (plasma) in different modes (see Table 1.) A - Accretor, E- Ejector, P Propeller, SE - SuperEjector, SP - SuperPropeller, SA - SuperAccretor, G - Georotator, M Magnetor. 
Table 1: Classification of neutron stars. $\mathrm{R}_{\mathrm{c}}$ - corotation radius, $\mathrm{R}_{\text {stop }}-$ stop radius, $\mathrm{R}_{\mathrm{G}}-$ gravitational capturing radius, $\mathrm{R}_{1}$ - light cylinder radius.

\begin{tabular}{|c|c|c|c|c|}
\hline Abbreviation & Type & $\begin{array}{l}\text { Characteristic } \\
\text { Radius Relation }\end{array}$ & Accretion Rate & $\begin{array}{l}\text { Observational } \\
\text { manifestations [16] }\end{array}$ \\
\hline $\mathbf{E}$ & Ejector & $\begin{array}{l}R_{\text {stop }}>R_{G} \\
R_{\text {stop }}>R_{1}\end{array}$ & $\dot{M}_{c} \leq \dot{M}_{c r}$ & $\begin{array}{l}\text { Radio pulsars, } \\
\text { Soft } \gamma \text {-repeaters, } \\
\text { Cyg X-3? } \\
\text { LSI+61 303? }\end{array}$ \\
\hline $\mathbf{P}$ & Propeller & $\begin{array}{l}\mathrm{R}_{\mathrm{c}}<\mathrm{R}_{\text {stop }} \\
\mathrm{R}_{\text {stop }} \leq \mathrm{R}_{\mathrm{G}} \\
\mathrm{R}_{\text {stop }} \leq \mathrm{R}_{1}\end{array}$ & $\dot{M}_{c} \leq \dot{M}_{c r}$ & $\begin{array}{l}\text { X-ray transients? } \\
\text { Rapid burster? }\end{array}$ \\
\hline $\mathbf{A}$ & Accretor & $\begin{array}{l}\mathrm{R}_{\text {stop }} \leq \mathrm{R}_{\mathrm{G}} \\
\mathrm{R}_{\text {stop }} \leq \mathrm{R}_{1}\end{array}$ & $\dot{M}_{c} \leq \dot{M}_{c r}$ & $\begin{array}{l}\text { Magnetic Ap-stars, } \\
\text { X-ray pulsars, Bursters, } \\
\text { Cataclysmic variables, } \\
\text { Intermediate polars }\end{array}$ \\
\hline $\mathbf{G}$ & Georotator & $\begin{array}{l}\mathrm{R}_{\mathrm{G}}<\mathrm{R}_{\text {stop }} \\
\mathrm{R}_{\text {stop }} \leq \mathrm{R}_{\mathrm{c}}\end{array}$ & $\dot{M}_{c} \leq \dot{M}_{c r}$ & $\begin{array}{l}\text { Earth, } \\
\text { Jupiter }\end{array}$ \\
\hline $\mathbf{M}$ & Magnetor & $\mathrm{R}_{\text {stop }}>\mathrm{a}$ & $\dot{M}_{c} \leq \dot{M}_{c r}$ & $\begin{array}{l}\text { AM Her, } \\
\text { polars }\end{array}$ \\
\hline SE & $\begin{array}{l}\text { Super- } \\
\text { Ejector }\end{array}$ & $\mathrm{R}_{\text {stop }}>\mathrm{R}_{1}$ & $\dot{M}_{c}>\dot{M}_{c r}$ & $?$ \\
\hline SP & $\begin{array}{l}\text { Super- } \\
\text { Propeller }\end{array}$ & $\begin{array}{l}\mathrm{R}_{\mathrm{c}}<\mathrm{R}_{\text {stop }} \\
R_{s t} \leq R_{l} \mathrm{R}_{\text {stop }} \\
\leq \mathrm{R}_{1}\end{array}$ & $\dot{M}_{c}>\dot{M}_{c r}$ & $?$ \\
\hline SA & $\begin{array}{l}\text { Super- } \\
\text { Accretor }\end{array}$ & $\begin{array}{l}R_{s t} \leq R_{e} \\
R_{s t} \leq R_{G}\end{array}$ & $\dot{M}_{c}>\dot{M}_{c r}$ & $\begin{array}{l}\text { SS433? } \\
\text { T Tau stars? } \\
\text { Ultra soft super luminous } \\
\text { sources? }\end{array}$ \\
\hline
\end{tabular}

The stage of GMR is most sensitive to period gravimagnetic parameter (cf [19],[75], [15]):

$$
y=\dot{M}_{c} / \mu^{2}
$$

Here, $\dot{\mathbf{M}}$ is the potential accretion rate on the magnetic rotator, defined by the Hoyle formula $\dot{M} \equiv \pi \boldsymbol{R}_{G}^{2} \boldsymbol{\rho} \boldsymbol{v}_{\infty}$ 


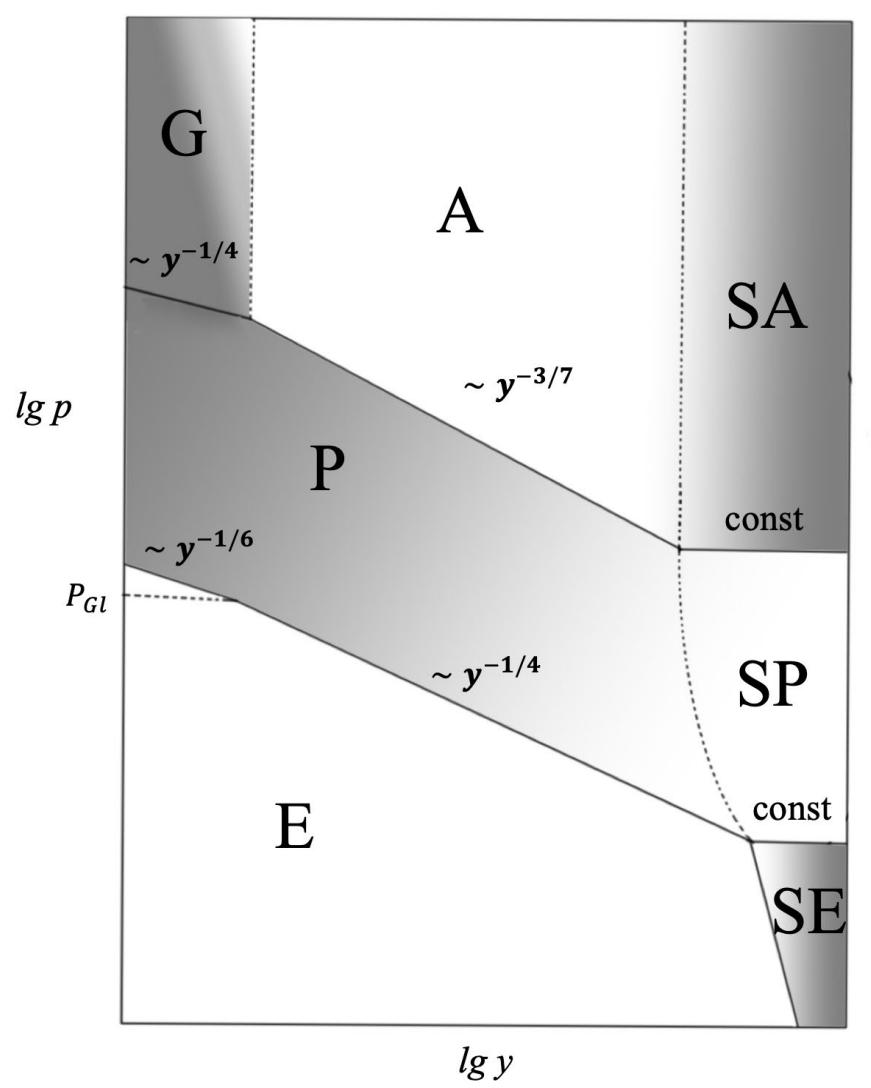

Figure 3.Qualitative p-Y diagram for gravimagnetic rotators calculated by Lipunov in 1987 $[15,16]$.

Thus a gravimagnetic rotator is an object with a magnetic dipole moment $\boldsymbol{\mu}$ and angular moment $\boldsymbol{K}=\boldsymbol{I} \boldsymbol{\omega}$, where $\boldsymbol{\omega}=\mathbf{2 \pi} / \boldsymbol{P}$ is the circular frequency of rotation, $\boldsymbol{P}$ is the orbital period; and with mass $\mathrm{M}$ (Fig. 1). Astrophysical manifestations of a magnetic rotator are defined by physical characteristics of the environment in which the rotator is placed. As previously shown, the state of the rotator mainly depends on the so-called gravimagnetic parameter (by Davies and Pringle in $1980 / 81[19,75]$ and by Lipunov in 1987 [15]) 


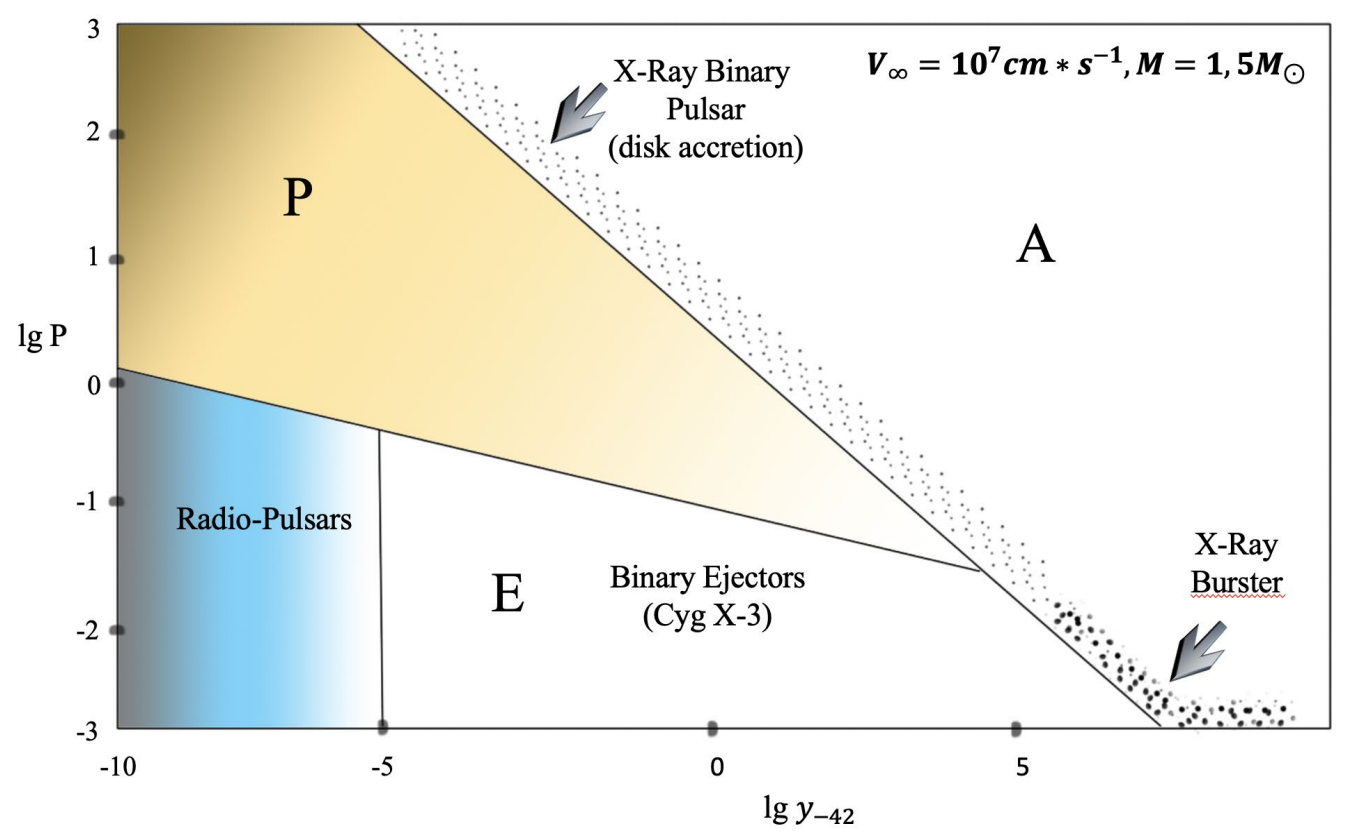

Figure 4. The "p - Y" diagram for neutron stars (under-critical regime) calculated by Lipunov in $1987[15]$.

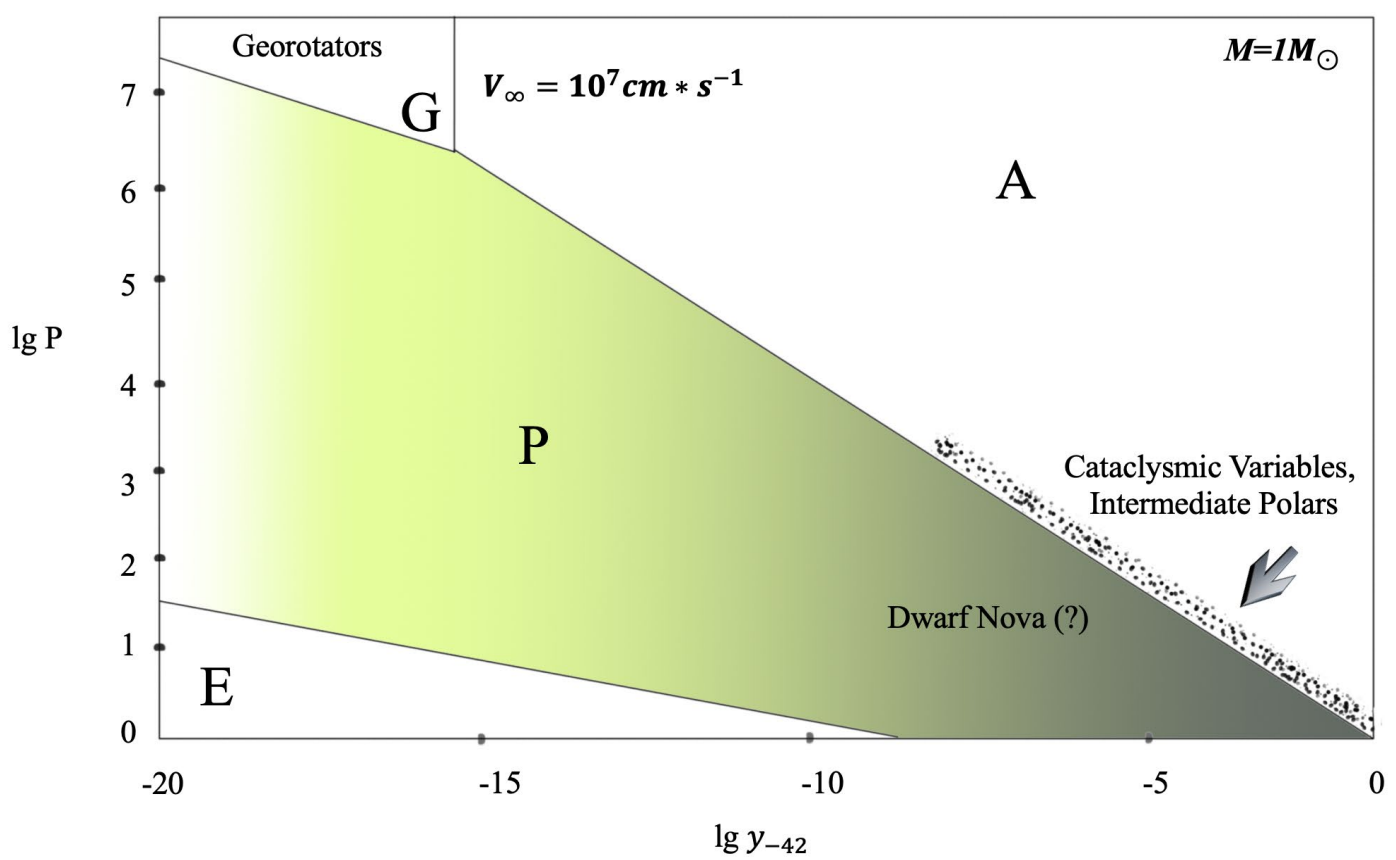

Figure 5. The "p - Y" diagram for white dwarfs calculated by Lipunov in 1987[15].

These diagrams were presented 30 years ago and we will check how relevant they are at present.

From the point of view of the GMR model, there is no fundamental difference between neutron stars and white dwarfs. In the case of disk accretion, both are "pressed" to the so-called 
equilibrium line, which corresponds to the condition $\boldsymbol{R}_{\boldsymbol{A}} \approx \boldsymbol{R} \boldsymbol{c}$ (where $R_{-} c$ is the corotational radius).

The $\mathrm{p}-\mathrm{Y}$ diagrams are convenient for representing the evolutionary relationship between different types of Gravimagnetic Rotators.

The period-gravimagnetic parameter diagram for NS in binary systems. (a) with NS magnetic field decay (the oblique part of the track corresponds to "movement" of the accreting NS along the so-called "spin-up" line), (b) a typical track of a NS without field decay in a massive binary system, calculated by Lipunov,Postnov, Prokhorov in 1996 [20].

These diagrams were presented several decades ago and in this report we will show how they look at the current level of observation data in 2018.

The equations of the evolution of compact stars as gravimagnetic rotators are described by Lipunov in 1987 [15].

$$
d I \omega / d t=K^{+}-K^{-}(4)
$$

Here $\boldsymbol{K}^{+-}$is the accelerating moment of forces, which depends on the accretion mode (disk accretion or wind-fed accretion), and the decelerating moment of forces in its most general form can be written as:

$$
K^{-}=-\varkappa_{t} \mu^{2} / R_{t}^{3}
$$

where $\boldsymbol{x}_{\boldsymbol{t}}$ and $\boldsymbol{R}_{\boldsymbol{t}}$ are the dimensionless factor and characteristic radius of interaction, respectively (see Lipunov's monograph [16]).The ecology of rotators involves this classification concerning their interaction with the environment and the determination of evolutionary laws. Possible implications for some particular astronomical objects are briefly described: X-ray and radio pulsars, single white dwarfs, X-ray bursters, cataclysmic variables, magnetic stars etc. (see also[16]).

The main observational properties of compact stars were shown to depend on the two parameters - $\boldsymbol{P}$ (or angular velocity $\omega=2 \boldsymbol{p i} / \boldsymbol{P}$ ), and gravimagentic parameter $\boldsymbol{y}=\dot{\boldsymbol{M}}_{\boldsymbol{c}} / \boldsymbol{\mu}^{2}$ (see Fig.1). Thus this single diagram can represent neutron stars and white dwarfs as X-ray pulsars, radio pulsars, $\mathrm{CV}$, intermediate polars, etc.. 


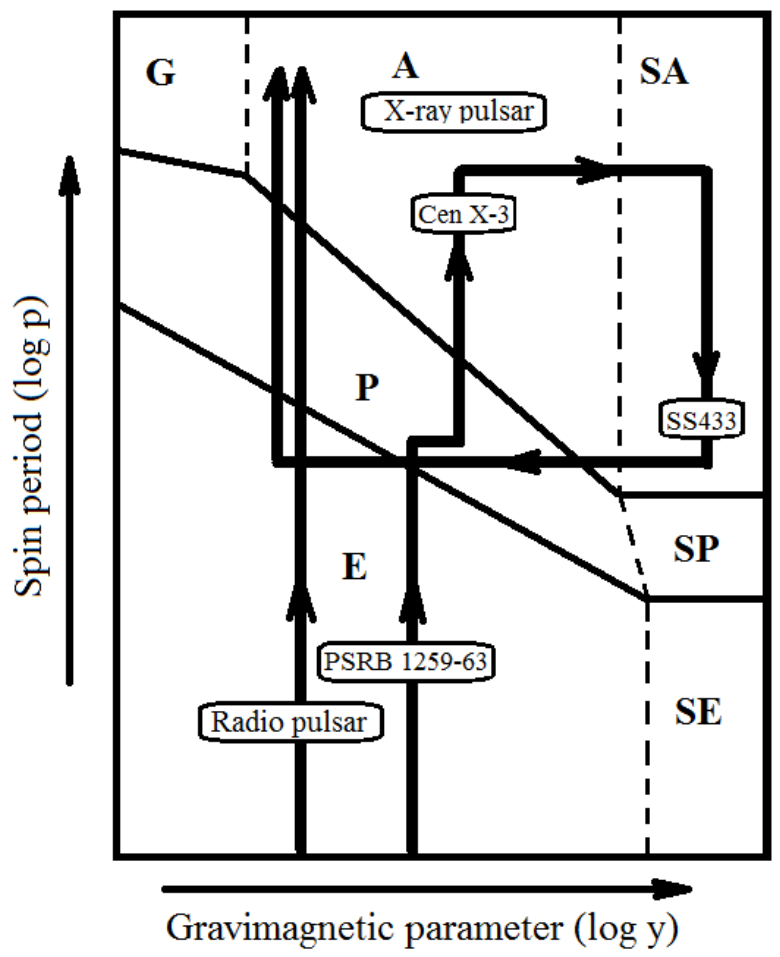

Figure 6.Evolution of magnetic neutron stars on the $\mathrm{p}-\mathrm{Y}$ diagrams. Qualitative picture.

\section{Scenario Machine Project.}

Half of all stars in our galaxy are binary. Of these, about half are close binaries. However, in the early 1980s the general laws of the evolution of normal binary stars were formulated by Crowford in 1955 [21], Snegko in 1967 [22], van den Heuvel in 1972 [23], and Tutukov, Yungelson in 1973 [24] on the one hand, and a common (albeit approximate) picture of the evolution of magnetized compact stars emerged where these objects formed at the end of thermonuclear evolution. The concept of magnetized neutron stars and white dwarfs as magnetic rotators by Swartzman in 1970 [13], by Illarionov\&Sunyaev in 1974 [25]; by Bisnovatiy-Kogan,Komberg in 1975 [26]; by xLipunov in1982[14] allowed for the first time to formulate the laws of their evolution. Of course, it was (and still is) an approximate theory of the evolution of ordinary and compact stars in binary systems. Therefore, as a "theory", some plausible evolution scenarios for the evolution of binary systems, including relativistic stages, were formulated, and population-based synthesis of various types of binary stars with compact components was carried out. The first such computer code was the Scenario Machine, which was developed in 1983 by Kornilov and Lipunov [27], [28], [29]; a description of its latest version can be found [30]) 


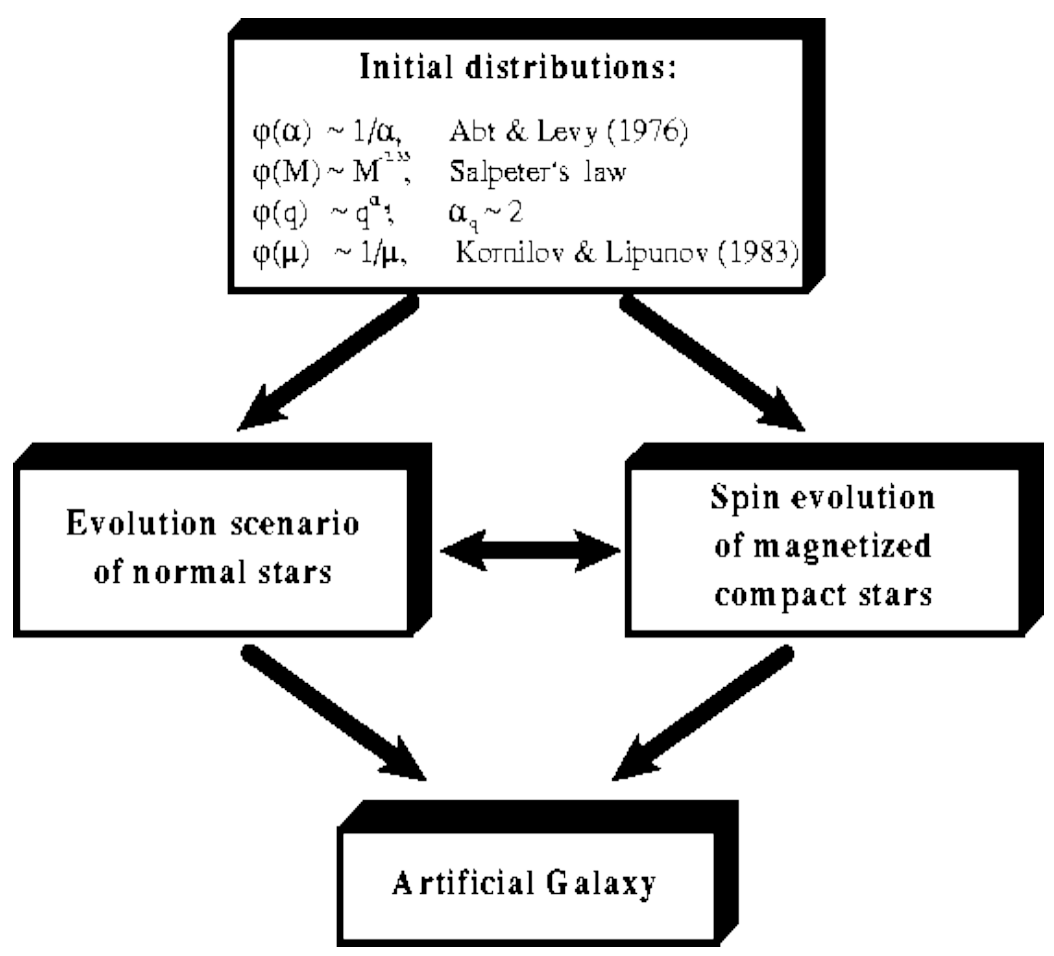

Figure 7: Schematic block diagram of the Scenario Machine.

\section{Population synthesis of GMR in massive binaries.}

The very first calculations showed an amazing variety of types of binary systems with compact stars (Table 2). It presents possible combinations of the states of the normal and relativistic components in a binary system. Four states of a normal star are successively presented in different columns: Main Sequence Star, Giant star, star filling its Roche lobe, and an actually compact star into which the normal star turns after passing through the first four stages in a binary system. We have included three possible products of the evolution of normal stars to save space: NS - neutron star or WD - white dwarf or black hole - BH. Vertically downwards are shown the stages of a neutron star a gravimagnetic rotator. In some cells of the state matrix, observational examples of corresponding binary systems are presented. As we see, already the first calculations of the Scenario Machine by Kornilov and Lipunov in 1983 [27,28] predicted a huge number of then undiscovered objects in the world of double stars (indicated by a question mark) - see Table 2. 
Table 2.

\begin{tabular}{|c|c|c|c|c|c|}
\hline \multicolumn{6}{|c|}{1983} \\
\hline $\begin{array}{l}\text { Evolution } \\
\text { Stage } \\
\text { of primary } \\
\text { and secondary } \\
\text { stars }\end{array}$ & $\begin{array}{l}\text { Main } \\
\text { Sequence }\end{array}$ & Giant & $\begin{array}{l}\text { Roche Lobe } \\
\text { Over flow }\end{array}$ & WR & Compact \\
\hline $\mathbf{E}$ & ? & $?$ & $?$ & $?$ & PSR 1913+16 \\
\hline $\mathbf{P}$ & $?$ & ? & $?$ & ? & $?$ \\
\hline $\mathbf{A}$ & $A 0535+26$ & $\begin{array}{l}\text { Vela X- } \\
1\end{array}$ & Her X-1 & $\begin{array}{l}\text { Close to } \\
\text { zero }\end{array}$ & ? \\
\hline SE & No & No & $?$ & No & ? \\
\hline SP & No & No & $?$ & No & $?$ \\
\hline SA & No & No & SS433? & No & ? \\
\hline BH & ? & $\begin{array}{l}\text { Cyg X- } \\
1\end{array}$ & SS433? & $?$ & ? \\
\hline
\end{tabular}

And in Table 3 we present the current situation 30 years after our prediction.

\section{The state matrix after 35 years of experimental research.}

However, after 35 years the same table was supplemented with the following objects, which we will consider in more detail below:

1.PSRB1259-63 ( Ejector + Main Sequence) - Radiopulsar with blue Be-stars .

2.X0331+53 (Propeller + MainSequence) -

3.ULXs Pulsars ( SA + OverflowRoche Lobe)

4.ULXs SS433 ( BH + Overflow Roche Lobe)

5.J07373039B ( P + Compact)

6.XTEJ0929-314 ( A + Compact)

7.GW150914 ( BH + Compact) 
Table 3:

\begin{tabular}{|c|c|c|c|c|c|}
\hline \multicolumn{6}{|c|}{2018} \\
\hline $\begin{array}{l}\text { Evolution } \\
\text { Stage of } \\
\text { primary and } \\
\text { secondary } \\
\text { stars }\end{array}$ & Main Sequence & Giant & $\begin{array}{l}\text { Roche Lobe } \\
\text { Overflow }\end{array}$ & WR & Compact \\
\hline $\mathbf{E}$ & $\begin{array}{l}\text { PSRB 1259-63 } \\
\text { Discovered in } 1992 \\
{[31]}\end{array}$ & ? & $?$ & $?$ & $\begin{array}{l}\text { PSR } \\
1913+16\end{array}$ \\
\hline $\mathbf{P}$ & $\mathrm{X} 0331+53$ & $?$ & $?$ & $?$ & $\begin{array}{l}\text { J07373039 } \\
\text { B }\end{array}$ \\
\hline $\mathbf{A}$ & $?$ & $\begin{array}{l}\text { Vela X-1 } \\
\text { Cen X-3 }\end{array}$ & $\begin{array}{l}\text { Her X-1 } \\
\text { Pulsating } \\
\text { ULXs }\end{array}$ & $?$ & $\begin{array}{l}\text { XTEJ0929 } \\
\mathbf{- 3 1 4}\end{array}$ \\
\hline SE & No & No & $?$ & No & $?$ \\
\hline SP & No & No & $\begin{array}{l}\text { ULX } \\
\text { discovered in } \\
2017[32]\end{array}$ & No & $?$ \\
\hline SA & No & No & ULXs & No & $?$ \\
\hline BH & $\begin{array}{l}\text { Be star MWC } 65 \\
\text { discovered in } 2014 \\
{[33]}\end{array}$ & CygX-1 & $\begin{array}{l}\text { SS433? } \\
\text { ULXs (?) }\end{array}$ & $?$ & $\begin{array}{l}\text { GW15091 } \\
4\end{array}$ \\
\hline
\end{tabular}

\section{White dwarfs as Gravimagnetic Rotators}

The study of magnetized white dwarfs in binary systems began at the beginning of the $1950 \mathrm{~s}$, when the first pulsating source was found associated with the dwarf nova DQ Her by Walker, M.F. 1958 [34]. Currently, we have information about dozens of such systems, which are often called Intermediate Polars. In our classification, these are systems similar to accreting X-ray pulsars, with the only difference that instead of a neutron star, as in the HZ Her / Her X-1 system, they contain b-magnetized white dwarfs. As a rule, normal stars in such systems are low-mass red stars (dwarfs) that fill their Roche lobe.

Among all possible products of the nuclear evolution of stars, white dwarfs are the most common objects in our Universe. It's all a matter of the Salpeter function - the mass distribution functions of the emerging stars growing toward small masses $\boldsymbol{\varphi} \sim \boldsymbol{M}^{-\alpha}(\alpha>1)$. It is the low-mass 
stars with $\boldsymbol{M}<\mathbf{8 - 1 0} \boldsymbol{M}_{\text {sun }}$ that are the progenitors (ancestors) of white dwarfs. So, in our Galaxy the number of neutron stars and black holes does not exceed 3\% that of white dwarfs, which is estimated at $\mathbf{1 0} \mathbf{1 1}^{11} \mathrm{M}_{\odot}$ by Lipunov et al. in 1995[35]. About half of all white dwarfs are in binary systems.

Low-mass binaries with white dwarfs are experimentally most well-studied and most common variable stars, forming an extensive class of supernovae, novae, dwarf novae, and ordinary cataclysmic stars. An analysis of numerous observations showed that, in addition to fast rotation, the $\mathrm{BC}$ can have magnetic fields whose intensity may reach several hundred mega gauss for a few seconds. These facts were established by the 1980s, when it became clear that rotation and magnetic field played as important part in the life of white dwarfs as in that of neutron stars. In particular, the assumption arose that white dwarfs with certain speeds of rotation and magnetic field strength values, as well as certain values of the parameters of the surrounding plasma, can manifest themselves as pulsating X-ray neutron stars or radio pulsars. This hypothesis naturally followed from the general approach to white dwarfs and neutron stars as magnetized gravitating, rotating bodies - gravity-magnetic rotators, introduced by Lipunov in 1987 [15]. The idea of this approach goes back to the works of Shvartsman [13], Illarionov, Syunyaev [25]; Bisnovaty-Kogan and Komberg [26] and consists in the fact that the physical state of the rotator and its evolution are determined by the ratio of gravitational and electromagnetic forces.

We call this object potential, because it becomes real only in the Accretor mode (A), when the rotating magnetic field of the rotator does not prevent the substance from falling onto the surface of the rotator. Obviously, in a dense medium with a slow-moving rotator, the accretion rate is large and the parameter y grows, but in highly magnetized rotators, the gravity-magnetic parameter can be small even in a dense environment. Single white dwarfs are of four types: Ejectors(E), Propellers(P), Accretors (A), and Georotators(G). The physical meaning of these stages is most easily demonstrated by the example of the evolution of a single magnetized white dwarf born with a fairly fast rotation (see Fig. 2). The density of the interstellar medium is low and even a white dwarf with weak magnetic field is at the ejection stage (E). In this case (E) accretion is impossible because it is prevented by the high pressure of Alfven waves and the relativistic stellar wind, so that the surrounding plasma does not even fall under the light cylinder whose radius determines the rotation frequency $\boldsymbol{R}_{\boldsymbol{l}}=\mathbf{c} / \boldsymbol{\omega}$. Like a neutron-star Ejector, a white-dwarf Ejector could manifest itself as a radio pulsar. The ejector spends the rotational energy that the white dwarf acquired at the stage of compression of the core of the ordinary star, after exhausting thermonuclear fuel reserves. In this case, the torque drops according to the equation (see Lipunov 1992 [16])

$$
d I \omega / d t=-\mu^{2} / R_{l}^{3}
$$

When the white dwarf slows down, the pressure of the relativistic wind decreases and the accreting plasma penetrates the light cylinder and the propeller stage begins. However, in the rapidly rotating quasi-static magnetic field of the white dwarf the radius of the $\boldsymbol{R}_{\boldsymbol{A}}$ (Alfven radius) magnetosphere, at the same time, exceeds the corotation radius, $\boldsymbol{R}_{\boldsymbol{A}}>>\boldsymbol{R}_{c}$, and the white dwarf continues to slow down. The propeller stage (P) for WD was predicted by Lipunov in 1987 [15] and appears to be observed in the AE Aquarii binary system, discovered by Schenker 
et al. in 2002 [36]. The total propeller energy is on the order of the power of gravitational energy release on the magnetosphere $\boldsymbol{L} \sim \dot{\boldsymbol{M}} \boldsymbol{G M} / \boldsymbol{R}_{\boldsymbol{A}}$. Deceleration continues and the accretion stage begins when the plasma completely reaches the surface of the white dwarf and its luminosity becomes $\boldsymbol{L} \sim \dot{\boldsymbol{M}} \boldsymbol{G} \boldsymbol{M} / \boldsymbol{R}_{\boldsymbol{W D}}$. However, for single white dwarfs with abnormally strong magnetic fields accretion is remains unaffected even when the rotation becomes very slow. The WD magnetosphere becomes similar to that of the Earth, and the accreting plasma flows around the magnetosphere like the solar wind, which does not hit the surface. Formally, this occurs when the radius of the gravitational capture becomes less than the radius of the magnetosphere: $\boldsymbol{R}_{\boldsymbol{A}} .>\boldsymbol{R}_{\boldsymbol{G}}$. We call such rotators Georotators (G). Here is the evolutionary track of a single white dwarf:

$\mathbf{E}=>\mathbf{P}=>$ Aor $\mathbf{E}=>\mathbf{P}=\mathbf{G}=>\mathbf{A}$

In binary systems, one more parameter is added: $\boldsymbol{a}$ (semimajor axis ). New stages are added. The trajectory of a binary system can be much more diverse (calculated by Lipunov in 1987[15], in 1988[37]; by Lipunov, Nazin in 1992[38]). White dwarfs-accretors are widely represented in observations, beginning with the first accreting dwarf DQ Her discovered in the early 1960-ies, as Walker found in 1958 [34], having a period of $\sim 60$ seconds.

The first population synthesis studies of low-mass binary stars made with the Scenario Machine in 1988 [37] by Lipunov and Postnov showed that the expected diversity of white dwarfs in different states is only slightly inferior to that of compact sources associated with neutron stars (Fig. 6, Table 3.).

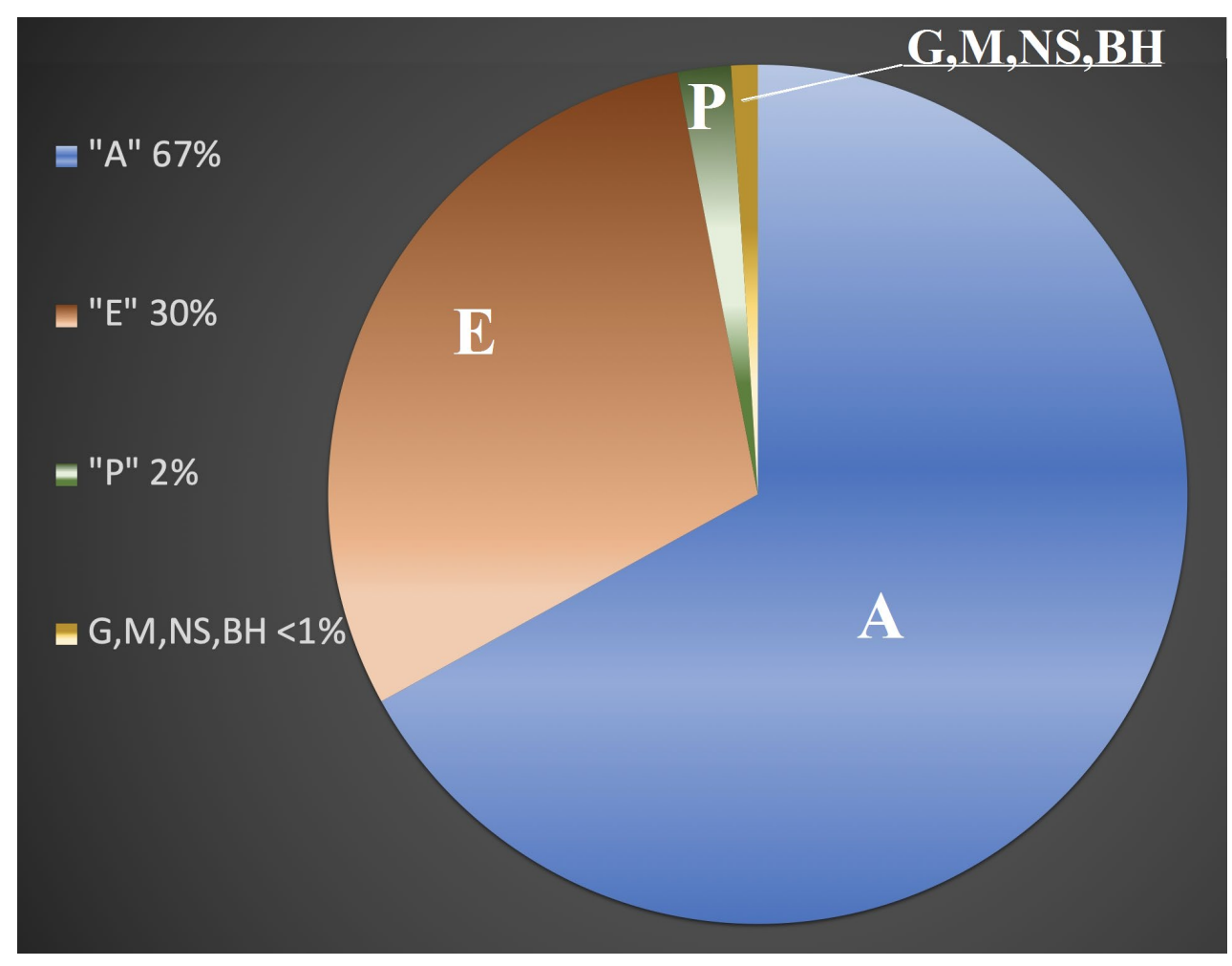

Figure 8: WD distribution among Gravimagnetic Rotator Types in low-mass binaries calculated by Lipunov and Postnov in 1988 [37]. 
Table 4: Matrix of states of binary systems with white dwarfs. No - the place, where there should not be such types.

\begin{tabular}{|l|l|l|l|l|l|}
\hline \multicolumn{5}{|c|}{ The 1987 situation as calculated by Lipunov,Postnov in [37] } \\
\hline & $\begin{array}{l}\text { Main } \\
\text { Sequence }\end{array}$ & Giant & $\begin{array}{l}\text { Roche Lobe } \\
\text { Overflow }\end{array}$ & WR & Compact \\
\hline E & Yes & Yes & Yes & Yes & Yes \\
\hline P & Yes & Yes & Yes & Yes & Yes \\
\hline A & Yes & $\begin{array}{l}\text { Symbiotic } \\
\text { Stars }\end{array}$ & DQ Her & No & Yes \\
\hline M & No & No & AM Her & No & No \\
\hline G & Yes & Yes & No & No & Yes \\
\hline
\end{tabular}

\section{The discovery of the first Ejector on a white dwarf in the AR Sco system.}

The first calculations of the number of ejecting white dwarfs in binary systems showed that it is comparable to the number of accreting WDs widely represented among cataclysmic variables and exceeds the number of another common type of white dwarfs - Propellers, calculated by Lipunov, Postnov in 1988 [37]. The reason for this lies in the fact that WDs have significantly higher moments of inertia and the duration of their ejecting stage is determined by the time and deceleration according to the magnetic dipole law:

$$
t=(d P / d t) / 2 P \approx 1.510^{11} \mathrm{mR}_{00.1}^{-4} B_{7}^{-2} P_{100}^{2} y r s(8)
$$

and the maximum period of WD, when the pressure of the surrounding plasma at the radius of the gravitational capture becomes greater than the pressure of the relativistic wind from WD is:

$$
P_{\max }<P_{E} \approx 200 B_{7}{ }^{1 / 2} R_{00.1}{ }^{3 / 2} \mathbf{v}_{6}{ }^{1 / 2} \rho_{-24}{ }^{-1 / 4} \mathrm{~m}^{-1 / 2} \mathrm{~s} \approx 200 \quad \mathrm{Y}_{42}{ }^{-1 / 4} \quad \mathrm{~s}
$$

Thus, the total time of the ejecting stage is equal to:

$$
t_{E} \approx 410^{11} B_{7}^{-1} R_{00.1}^{-1} v_{6}^{-1 / 4} \dot{M}-10{ }^{-1 / 4} y r s(10)
$$

Thus, single white dwarfs remain ejectors during their entire lifetime, if their field does not exceed tens of mega gauss.

In binary systems during the accretion stage, the rotation of the white dwarf is possible and, as a result, the implementation of the so-called " Recycled pulsars scenario " , calculated by 
Lipunov,Nazin in 1992 [38]). The fact is that during disk accretion (which is realized in lowmass binary systems known as intermediate polars) on a magnetized compact object, its rotational evolution leads to the so-called equilibrium state, when the accelerating moments of forces associated with the angular momentum of the accreting mass is balanced by the decelerating moment of forces due to the interaction of the magnetic field with the plasma located beyond the corotation radius.

$d I \omega / d t=\dot{M} \sqrt{ }\left(G M R_{A}\right)-\varkappa_{t} \mu^{2} / R_{c}^{3}=0$,

where $\boldsymbol{R}_{A}$ is the Alfven radius at which the gravitational energy equals the magnetic energy of the plasma; $\boldsymbol{x}_{t}$ is a dimensionless factor, and $\boldsymbol{R} \boldsymbol{c}=\left(\boldsymbol{G M} / \boldsymbol{\omega}^{2}(\mathbf{1} / \mathbf{3})\right)$ - is the corotation radius where the rotation speed of the magnetic field lines is equal to the Keplerian rotation speed of the plasma in the disk.

Thus, accretion in binary systems can accelerate or maintain the fast rotation of the compact magnetized star. After the end of accretion, the rotation period of the white dwarf period may be less than the critical $\boldsymbol{P}_{\boldsymbol{E}}$ (formula (5)) and the white dwarf is again at the Ejection stage.

The discovery of the radio pulsar shows that ejecting white dwarfs not only exist, but because of their properties they also strongly resemble classical radio pulsars on neutron stars.

In this case, it can be expected that the acceleration of relativistic particles occurs near the magnetic poles, where the electric and magnetic fields are parallel. The absolute upper limit of the energy to which elementary particles can be accelerated under such conditions is obviously equal to:

$\mathrm{E}_{\max }=\mathrm{e} \mathbf{E} \mathrm{R}=\mathrm{e}(\mathrm{v} / \mathrm{c}) \mathrm{B} \mathrm{R}=\mathrm{e}(\omega R / c) B R \approx 1.510^{16} P_{100}{ }^{-1} R_{5000} B_{8} \mathrm{eV}(12)$

Where $\mathbf{P}_{100}=\boldsymbol{P} / \mathbf{1 0 0 s}, \boldsymbol{R}_{5000}=\mathbf{R} / \mathbf{5 0 0 0} \mathrm{km}-$ white dwarf radius, $\boldsymbol{B}_{8}=\boldsymbol{B} / \mathbf{1 0} 0^{8} \mathbf{G}$.

Of course, in reality, acceleration apparently occurs in a small gap at the poles, and the actual energy of the particles is evidently lower. However, the limit is so high that we see no fundamental obstacles preventing a white dwarf with a several hundred megagauss strong magnetic field from accelerating ultrahigh-energy particles.

The recent discovery of a radio pulsar in a system consisting of two M-type dwarfs and a degenerate white dwarf (discovered by Marsh et al. in 2016 [39]) not only confirmed our understanding of the evolution of compact stars (neutron stars and white dwarfs), but also raised unexpected questions about white dwarfs as sources of cosmic rays (see a discussion by Lipunov et al. in 2016 [40]).

Polarimetric evidence of a white dwarf pulsar (Polarization 1.97 min Spin Variability) in the binary system AR Scorpii (see Buckley et al. [41]) provides direct evidence that in this case we are dealing with a complete analogue of radio pulsars on neutron stars!

Table 5: Matrix of states of binary systems with white dwarfs. Question marks stand at undiscovered stages. No - where there should not be such types. 


\begin{tabular}{|l|l|l|l|l|l|}
\hline \multicolumn{5}{|c|}{ 2016First Ejector WD discovery Marsh et al. Nature, 537, 374 } \\
\hline & $\begin{array}{l}\text { Main } \\
\text { Sequence }\end{array}$ & Giant & $\begin{array}{l}\text { Roche } \\
\text { LobeOverflow }\end{array}$ & WR & Compact \\
\hline E & AR Sco & Yes & Yes & Yes & Yes \\
\hline P & Yes & Yes & Yes & Yes & Yes \\
\hline A & Yes & Symbiotic & DQ Her & No & Yes \\
\hline M & No & No & AM Her & No & No \\
\hline G & Yes & Yes & No & No & Yes \\
\hline
\end{tabular}

\section{The Universal p-Y diagram after 30 year.}

Here we demonstrate the versatility of the model of a gravity magnetic rotator in the light of the discoveries made over the past three decades. Figure 10 presents the observational parameters of 8 types of astrophysical sources in the model of a gravity-magnetic rotator. We make one important remark. We do not show in the figure supercritical regimes and georotator mode. First, the position of these types of a geotor depends on an additional parameter - the magnetic dipole moment. Second, we did not clutter the diagram with supercritical modes of interaction of a gravitating magnetized star with the surrounding plasma since because there is no really confirmed observational candidates (see, however, Erkut, M. Hakan discovery in 2017 [32], which proposes a superpropeller model for explaining non-pulsing ULX sources). For those reasons, we do not show the Georotator stage (G).

We emphasize that the $\mathrm{p}-\mathrm{Y}$ diagram is the only diagram that simultaneously shows not only various astrophysical objects associated with one particular type of compact star (for example, a neutron star), but also objects associated with various types of compact stars - white dwarfs and neutron stars. Let's make some explanations to the universal diagram presented here. X-ray pulsars and intermediate polarw, as expected, fall into the region of the accretor. Obviously, to determine the $\mathrm{Y}$ parameter you need to know the accretion rate and the magnetic dipole moment. The accretion rate is determined from the observed X-ray luminosity $\boldsymbol{L}_{\boldsymbol{x}}=\dot{\boldsymbol{M}} \boldsymbol{G M} / \boldsymbol{R}_{\boldsymbol{x}}$. The radius and mass of the neutron star is everywhere assumed to be $\mathbf{1 0} \mathbf{~ k m}$ and $\mathbf{1 . 5} \mathbf{M}_{\boldsymbol{\Theta}}$, respectively, and the corresponding quantities for for white dwarfs, $5000 \mathbf{~ k m}$ and $1.5 \boldsymbol{M}_{\boldsymbol{\Theta}}$, respectively,

For intermediate polars, we determine the magnetic dipole moment from the observed cyclotron lines and spectral features (see Suleimanov et al. [42]) using the dipole formula $\boldsymbol{\mu}=\boldsymbol{B} \boldsymbol{R}_{\boldsymbol{x}}{ }^{3} \boldsymbol{2}$. For the same f simplicity, we adopt the same radius for all stars, which we set equal to $\mathbf{R x}=\mathbf{5 0 0 0}$ km . 
Let us look to the X-ray pulsars on neutron stars that have disk accretion realized from the condition that they are on average in equilibrium (calculated by Lipunov in 1987 [15] and in [16]):

$$
<d I \omega / d t>=<\dot{M} \sqrt{ } \boldsymbol{G M R} R_{A}-\mu^{2} / R_{c}^{3}>=0
$$

Or

$$
<\dot{M} \sqrt{ } G M R_{A}>=\mu^{2} \omega^{2} e q / G M
$$

From here we obtain the mean value of the gravimagnetic parameter $\mathbf{Y}$.

$$
<Y_{e q}>\approx 10^{-42} p^{6 / 7}(15)
$$

Of the three principal parameters of the quantities included in these equations, $\dot{M}, \omega$ and $\mu$. the most strongly varying quantity is $\dot{\boldsymbol{M}}$, so we can assume that $\omega_{e q}=2 \pi / \boldsymbol{p}_{\text {obs }}$ that is, $\boldsymbol{p} \approx \boldsymbol{p}_{\boldsymbol{o b s}}$, where $\boldsymbol{p}_{\boldsymbol{o b s}}$ is the pulsar period observed at a given time.

This technique works well for Her X-1 type systems in which optical stars - donors have an average mass and accretion rate changing only slightly so that the gravimagnetic parameter of the pulsar is near the equilibrium value $\left\langle\boldsymbol{Y}_{e q}\right\rangle$ most of the time. This is proved by the fact that the mean acceleration time of a pulsar $\left\langle\mathbf{t}_{\mathrm{su}}>\approx \mathbf{3 0 0} 000\right.$ years is much longer than the strong acceleration observed in episodes $\left\langle\mathbf{t}_{\mathrm{su}}>\right| \min \approx 20000$ years (calculated by Lipunov in 1987b [43]) and therefore the pulsar is in the so-called "catastrophic equilibrium"[43] (see the figure in page 147 of "The Astrophysics of Neutron Stars" by Lipunov, 1992 [16]).

If we turn to binaries with a red dwarf, this approach should already be used with caution. We are talking about donors with a mass of $\sim \mathbf{0 . 3 - 0 . 7 M _ { \boldsymbol { \Theta } }}$ with powerful convective layers below the surface. The mass flow in such systems is extremely unstable and the rate of accretion may vary by orders of magnitude. Such a situation arises for rapidly evolving neutron stars - X-ray bursters or systems of neutron stars close to the formation of millisecond radio pulsars. And the same picture arises for intermediate polars. In these cases, the average value of $\left\langle\boldsymbol{Y}_{e q}\right\rangle \sim\langle\dot{M}\rangle$ may differ by orders of magnitude from that observed at the times of X-ray activity. In fact, the average accretion rate will be lower and the compact star may spend in the Propeller (P) or even the Ejector (E) states most of the time. The P-Y diagram (Fig. 9) for these sources shows the locus corresponding to the episodes of X-ray activity. 


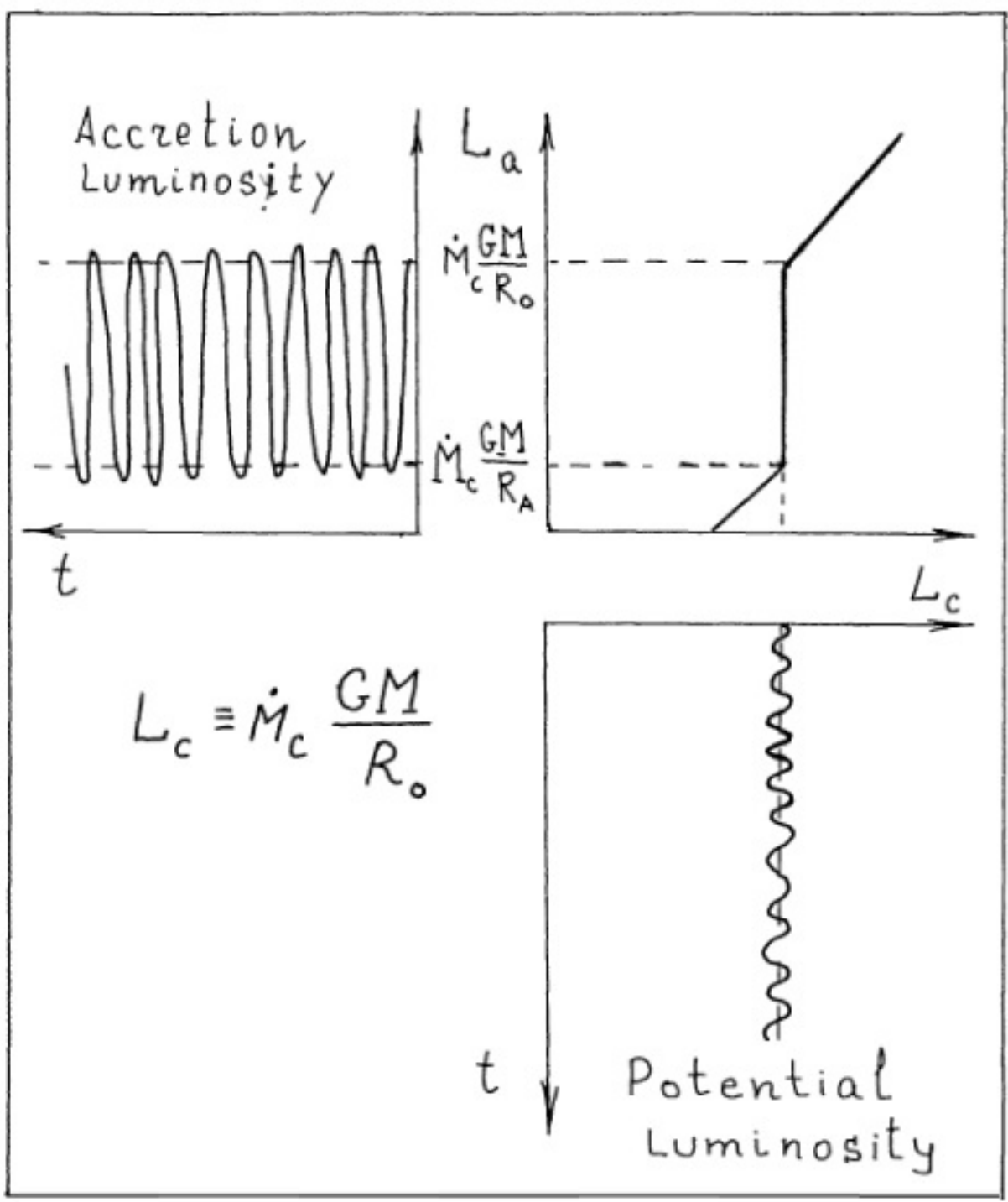

Figure 9: Catastrophic equilibrium: a weak change in the accretion rate leads to strong (2-3 orders of magnitude) change in the X-ray luminosity, calculated by Lipunov in $1987[15])$.

The situation is similar for those X-ray accreting pulsars, where the donor is a massive rapidly rotating non-stationary Be-type main sequence star. Here, huge oscillations of the accretion rate are associated with the nonstationary character of the mass loss of Betype star due to the rotational instability of its outer layers. Because of their rapid rotation, such stars are surrounded at the equator by expanding disc-shaped shells. Over several dozen years Be-type stars may remain in a calm state, interrupted by the release of a shell. To this is added the significant ellipticity of the orbits of neutron stars, which is another factor in the sharp variability of the accretion rate. The picture becomes truly chaotic, and for these X-ray pulsars we show the Y-parameter at the time of X-ray activity. Although in reality some of them, on average, are in a mixed $\mathbf{A} \leftrightarrow \mathbf{P}$ (AccretorPropeller) state ascalculated by Gnusareva, Lipunov in 1985 [44]. 


\section{Neutron Stars Radio pulsars.}

Neutron stars were discovered as radio pulsars by Hewish et al. in 1968 [3]. As should be the case, all radio pulsars on neutron stars fall within the Ejectors area (E). Most of them are single neutron stars or stars paired with degenerate companions - so the donor here is the interstellar medium for which the average parameters for the Galaxy are adopted. The magnetic field of radio pulsars was found by delay in view of the energy losses of radio pulsars can be described by the magnetic dipole formula: $\boldsymbol{\mu}_{30}$ $=10\left(p \dot{p}_{-15}\right)^{1 / 2}$, where the period is measured in seconds, and the derivative of the period is dimensionless $\dot{p}_{-15}=\dot{p} / 10^{-15}$.

All radio pulsars can be subdivided into two types - one (young neutron stars) are born initially with small periods, at least in binary systems. And others - old neutron stars unwind in the process of accretion in binary systems (recycled scenario by Alpar et al. in 1982 [45]). These pulsars represent two areas in our diagram,: the first - at the top left part of the Ejectors zone, and the second - mostly the millisecond pulsars - are located in the bottom right part of the diagram because of their weak magnetic fields. Here we pay attention to the upper envelope of radio pulsars - first, the envelope goes parallel to the line (PE) separating the Ejectors and Propellers. Physically, this is understandable the death line of radio pulsars on our diagram is parallel to the line $\boldsymbol{p} \sim \boldsymbol{Y}^{-1 / 4}$, and possible can be by itself the death line not only for ejectors, but also for radio pulsars (this was first noted by Dmitry Kolosov in 1989 [46]).

Moving further along the upper edge, we notice that the slope increases and becomes parallel to the line separating the Propellers (P) and Accretors (A). This is natural since millisecond pulsars are former old accreting stars lying on the equilibrium line of Lipunov (1987a [15]) (it was later called the Spin-Up Line).

\section{Magnetars. Anomal Pulsars (ASP) and soft gamma repeaters (SGR).}

The possibility of the existence of neutron stars with abnormally strong magnetic fields was first suggested by Nikolay Shakura in 1975 [47]. In this work Shakura pointed out that despite its average accretion rate, the X-ray pulsar Vela X-1 had an abnormally long period of $\sim 280$ seconds. For example, Her X-1, which has a similar luminosity, showed a gyroline corresponding to a magnetic field of $\sim 10{ }^{12} \mathrm{Gs}$. Considering that the corotation radius should be on the order of the Alfven radius $\boldsymbol{R}_{c} \approx \boldsymbol{R}_{A}$, he estimated the magnetic field strength to be $\sim 10^{14}$ Gs! Later, Lipunov and Shakura in 1976 [48] showed that other long-period Pulsars should be close to equilibrium, and this result was brilliantly confirmed when, in the next 5 years, X-ray accreting pulsars began to show not only acceleration, but also episodes of slowing rotation (1992 [16]). Lipunov in 1982c [49] used another method for measuring the magnetic field of accreting pulsars, namely, by determining the magnetic dipole moment of spindown pulsars. At the same 
time, the existence of abnormally strong magnetic fields in neutron stars was confirmed. Nevertheless, it should be emphasized that in systems without accretion disks (direct accretion from the stellar wind - the so-called wind fed accretion), the accelerating moments are small, and vice versa, the retarding moments can be much higher (in 1999 [50]). So that the question about existence remained open, the situation became much more complicated and the question remained open until now.

On March 5, 1979, the Soviet orbital gamma-ray satellite Venera-11 detected by KONUS detector a bright gamma-ray burst, which showed, along with a short bright flash, strictly periodic damped oscillations with a period of $\sim 8$ seconds (publishied by Mazets et al. in 1979 [51,52]). Hard spectrum with periodicity left no doubt that we were dealing with a magnetized neutron star. Later it turned out that similar neutron stars - anomalous X-ray pulsars (ejectors) - also show similar periods, and their period change corresponds to magnetic field strengths of $\mathbf{1 0}^{\mathbf{1 3}} \mathbf{- 1 0} \mathbf{1 0}^{\mathbf{1 5}} \mathbf{G}$ (in 2015 [53]).

We now know about 20 Magnetars - anomalous pulsars (ASP) and soft gamma repeaters (SGR - Soft Gamma Repeaters) presented on our p-Y diagram. The data about them are adopted from the work of Olausen, Kaspi $(2014[54,55])$.

All of them are in the top left part of the Ejectors area. 


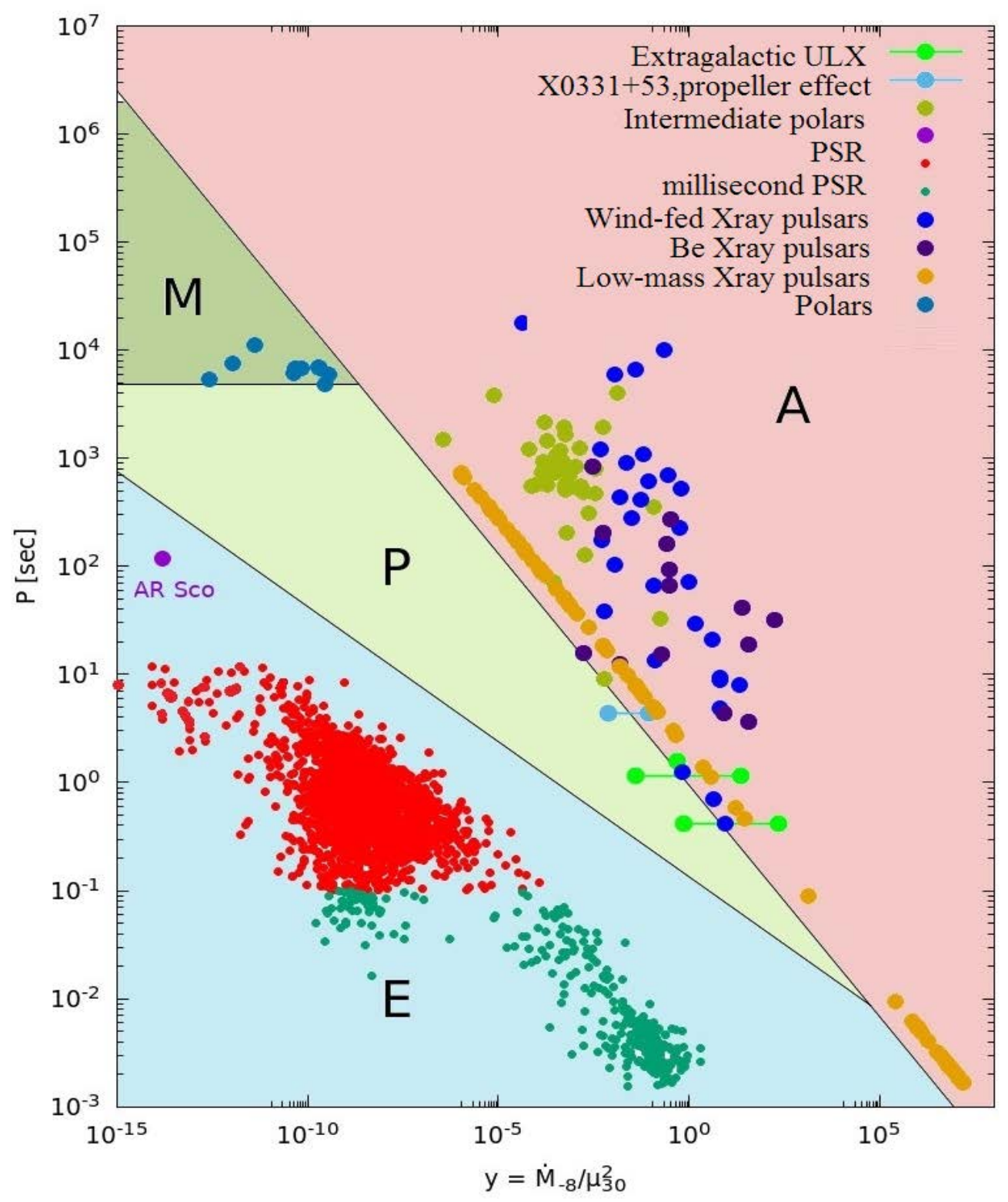

Figure 10: The universal diagram Period - Gravimagnetic rotator diagram for most of the observed types of neutron stars and white dwarfs. Intermediate Polars are shown in green; X-ray accreting pulsars in ochre; ULX pulsating sources in light green; radio pulsars on neutron stars are in red circle. Accreting X-ray Binary Pulsars were taken from http://www.iasfbo.inaf.it/ mauro/pulsar_list.html ; PSR from [56]; AR Sco from [39]; X0331 + 53, Propeller from [57]; Intermidiate Polars from [42]; Polars [58]; Triangles - Magnetars (Olausen and. Kaspi in2014 [54,55]) 


\section{Propellers}

The source X0331 $+53 \mathrm{~b}$, which shows episodes of the propeller mode, is depicted by two blue dots corresponding to the transitions of the neutron star from the Accretor state (A) to the propeller mode (P) and back. Note that this transition is accompanied by a catastrophic (approximately 100 times) decrease in luminosity with a gradual weak change in the potential accretion rate, for example, due to the changing distance from the donor star moving in an eccentric orbit. As noted for the first time by Gnusareva, Lipunov in 1985 [44], this change is due to the passage through the centrifugal barrier. From the $\mathbf{R}_{\mathbf{A}}<\mathbf{R c}$ state the rotator enters the $\mathbf{R}_{\mathbf{A}}>\mathbf{R c}$ state, and the accretion to the surface changes with the total luminosity of the accretor $\boldsymbol{L}(\boldsymbol{A})=\dot{\boldsymbol{M}}_{c} \boldsymbol{G} \boldsymbol{M} / \boldsymbol{R}_{\boldsymbol{0}}$ sharply (catastrophically) decreasing to the luminosity of the Propeller $\boldsymbol{L}(\boldsymbol{P})=\dot{\boldsymbol{M}}_{c} \boldsymbol{G} \boldsymbol{M} / \boldsymbol{R}_{\boldsymbol{A}}$. At the moment of the transition the accretion rate at the boundary of the magnetosphere changes smoothly, and therefore the magnitude of the jump (the amplitude of the X-ray $\operatorname{dip}$ (gap)) is: $\boldsymbol{L}(\boldsymbol{P}) / \boldsymbol{L}(\boldsymbol{A})=\boldsymbol{R}_{x} / \boldsymbol{R}_{\boldsymbol{A}}$, this is the radius of the surface of the star. However, at the time of the transition, the Alfven radius is approximately equal to the corotation radius, which is a function of the period of rotation of the rotator (!) $\boldsymbol{R}_{c}=\left(G M / \omega^{2}\right)^{1 / 3}$.

$$
L(\mathrm{P}) /\left(L(\mathrm{~A})=R_{x} / R_{c} \approx 10^{-2} p^{-2 / 3}(16)\right.
$$

In the last formula we adopt $\boldsymbol{R}_{\boldsymbol{x}}=\mathbf{1 0} \mathbf{~} \mathbf{~ m}$ and the period is in seconds!

\section{Black holes in binary systems with massive main-sequence stars}

In the world of binary stars with relativistic components, an old problem existed the lack of black holes paired with massive main-sequence stars (by Rappaport, van de Heuvel in 1982 [59], van de Heuvel ,Rappaport in 1982[60]) According to Lipunov and Karpov (2000 [61]), the lack of black holes with the standard blue maon-sequence stars is due to the fact that in these systems the optical companions lie deep inside their Roche lobes. As a result, the stellar wind velocity in the orbit is significantly higher than in the case of supergiants. The specific angular momentum of the matter captured by an accreting star is known to be proportional to the squared radius of gravitational capture $\left(\boldsymbol{R}_{\boldsymbol{G}}=\mathbf{2} \boldsymbol{G M} / \boldsymbol{V}_{\boldsymbol{w}}{ }^{2}\right)$, in detached systems it is too small to form an accretion disk. In addition to the magnetic pumping effect (see Lipunov 1992 [16]), this effect leads to the fact that in such systems spherically symmetric accretion without magnetic field is realized whose efficiency is millions of times lower than that of disk accretion. Thus, we simply cannot detect black holes in such systems. However, in systems with rapidly rotating Be-type stars, the donor supplies the mass with much lower velocity, permitting the formation of an accretion disk! Raguzova and Lipunov (1999) used the population synthesis method to demonstrate the possibility of the formation of such systems. A 
very likely candidate for this type of binary system was discovered by Casares with coauthors in 2014 [33] (see also recent work on this topic by Brown et al. [62]).

\section{Ultra luminous $x-$ ray pusars.}

The newcomers in this diagram are pulsating ULX sources. We emphasize that these sources are not supercracretors, because the luminosity of the accretion flow in the magnetosphere does not exceed the Eddington limit and the entire plasma successfully reaches the surface of the neutron star. As a result, the luminosity of such an x-ray pulsar can significantly exceed the Eddington limit and reach the observed values for extragalactic objects whose pulsations have been discovered in recent years by NGC 5907, NGC 7793 P13, M82 X-2.

Table.

\begin{tabular}{|l|l|l|l|l|}
\hline Galaxy & p (s) & L(erg/s) & Satelite & Reference \\
\hline NGC 5907 & 1.42 & $2.210^{41}$ & $\begin{array}{l}\text { Swift, XMM, } \\
\text { Nustar, } \\
\text { Chandra }\end{array}$ & $\begin{array}{l}\text { By Israel et al. } \\
\text { in 2017a[63] }\end{array}$ \\
\hline NGC 7793 & 0.42 & $1.610^{40}$ & $\begin{array}{l}\text { Einsten,XMM, } \\
\text { NuSTAR, Rosat }\end{array}$ & $\begin{array}{l}\text { By Israel et al. } \\
\text { in 2017b[64] }\end{array}$ \\
\hline M82 X-2 & $\mathbf{1 . 3 7}$ & $3.710^{40}$ & Nustar, Chandra & $\begin{array}{l}\text { By Bachetti et } \\
\text { al. in 2016 [65] }\end{array}$ \\
\hline
\end{tabular}

As noted in "Astrophysics of Neutron Stars" by Lipunov in 1992 [16], it is difficult to imagine another scenario in which the luminosity during stationary accretion on the magnetosphere would significantly exceed the Eddington limit and at the same time the source would be an X-ray pulsar. . This gives a natural limit to the accretion of subcritical regimes $\mathbf{A}$ :

$$
Y_{-42} \leq 10^{7} / \dot{M}_{18}
$$

Recall that $\boldsymbol{Y}_{-42}=\dot{M}_{18} / \boldsymbol{\mu}_{30}{ }^{2}$. Putting $\boldsymbol{L}_{\boldsymbol{x}}=10^{38} \dot{\boldsymbol{M}}_{18} \mathrm{erg} / \mathrm{s}$, we obtain that the condition of the subcritical mode of the accretor $\mathbf{A}$ can be written in the form:

$$
L_{x} \leq 310^{41} \mu_{30} \mathrm{erg} / \mathrm{s}
$$

At the magnetic poles of a neutron star, the luminosity can greatly exceed the Eddington limit. The radiation there can take on a sharply anisotropic character and mainly go across the accretion disk without touching the mass flows in the binary system, that is, without affecting the supercritical regime. In this case, the total luminosity of the pulsar can reach the value:

$$
L_{x}=L_{E d d}\left(R_{A} / R_{x}\right) \approx 10^{40} \mathrm{erg} / \mathrm{s} \quad Y_{-42}^{-2 / 7}
$$


$\mathbf{Y}_{-42}=\mathbf{Y} / \mathbf{1 0}^{-42}$ (CGS). The sources mentioned above are present in the universal diagram as Accretors (A), and not super accretors (SA).

This mode is accompanied by significant losses on neutrino radiation, since in the polar column the temperature can exceed $1 \mathrm{Mev}$. The process of electron-positron pair production with energy loss through electro-weak interaction (Zeldovich et al. in 1972 [66]):

$$
\gamma \rightarrow e^{+}+e^{-} \rightarrow v_{e}+v_{e}^{*}
$$

Such a scenario seems to be realized by ULX sources - pulsars, where the accretion rate can reach $10^{-6}-10^{-5} M_{\$} / y r$. This in turn gives rise to two new effects characteristic of these stages, which may be observed in the future (Lipunov 1992 [16]). First, since the luminosity of the polar column significantly exceeds the Eddington limit (Basko,Sunyaev in 1975[67]), the accretion in the polar column must be pulsating, and transient in nature at times (Lipunov, 1992 [16]):

$$
\boldsymbol{t} \sim \boldsymbol{R}_{A} / \sqrt{ }\left(\boldsymbol{G M x} / \boldsymbol{R}_{A}\right) \sim 210^{-2} \mathrm{Y}_{-42}^{3 / 2} \mathrm{~s}
$$

Well, in a very distant future, we can expect the discovery of a neutrino pulsar caused by the anisotropy of neutrino production for neutron stars with an anomalously strong magnetic field $B>\mathbf{4} 10^{13} \mathbf{G}$.

Returning to the X-ray sources in low-mass binaries, we note that they are located in the bottom right part of the Accretor region. These are old neutron stars that have practically lost their magnetic field and sped up to the smallest rotating periods known for objects in the Universe.

As for the ejectors, they are represented in the diagram by standard radio pulsars. The periods and dipole moments are adopted from the catalog [of??] Radio pulsars, and the accretion rate for all of them is supposed to be equal to the average value of $M=10^{10} \mathrm{~g}$ / s. The same applies to millisecond pulsars (the bottom right corner of the $\mathbf{E}$ zone), including binary radio pulsars with degenerate components, as well as to Magnetars. This is understandable, since the interstellar medium is the donor for all these objects. An exception is the radio pulsar in a binary system with a blue Be-type star PSRB 125963 (Johnston et al. in 1992 [31]).

The accretion rate is very difficult is very difficult to restore[estimate??] in this case. However, it is qualitatively clear that each orbital revolution in this system leads to a periodic change in the $\mathbf{Y}$ parameter, which we demonstrate with a horizontal segment.

Of particular interest is the "newcomer" among the ejectors of the radio pulsar - the white dwarf AR Sco! 


\section{Polars.}

Finally, we will look at the new class of these objects in the p-Y diagram. In the process of preparing this report, it became clear to us that Polars can be naturally shown on this diagram in a separate area - the field of Magnetors (M). This type of magnetosphere was proposed (by Mitrofanov et al. 1977 [68]) as accreting white dwarfs with magnetic fields so strong that the donor — an optical star — is inside the white dwarf's magnetosphere: $\boldsymbol{R}_{\boldsymbol{A}}>\boldsymbol{a}$. However, the Alfven radius is determined by the Y parameter, and the semi-axis is double, respectively.

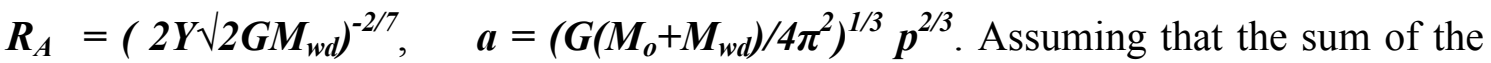
masses is characteristic of binary systems - it should be equal to 1 solar mass for a polar consisting of a red and white dwarf. Equating both values, we note that the critical line for Magnetors - that is, polars, is

$$
p_{M}>=2 \pi /\left(G M_{t o t}\right)^{-1 / 2} a^{3 / 2} \approx 10^{3} s\left(M_{t o t} / 0.1 M_{\Theta}\right)^{-1 / 2}\left(a / 0.1 R_{\Theta}\right) 3 / 2
$$

We drew a horizontal line for $\mathbf{M t o t}=\mathbf{0 . 5} \mathbf{M}_{\odot}, \mathbf{R}=\mathbf{0 . 1} \mathbf{R}_{\boldsymbol{\Theta}}$. However, unlike the situation with Accretors, the Magnetor period is determined by the magnetic synchronization of white and red dwarfs and is exactly equal to the orbital period of the binary system. In other words, Magnetors - Polars should line up along the pM line, which is what happens in the diagram. The values of magnetic fields and accretion rate are taken from Cherepashchuk et. al. in 1996 [58].

\section{Conclusion}

We mainly focused on the most well-studied magnetized astrophysical objects for which the Gravity Magnetic Rotator model is fundamental. These are primarily neutron stars and white dwarfs. Here we have not touched upon more exotic objects whose existence in our opinion is likely. These are the so-called Spinars (introduced by Lipunov in 1987 [15]). Such objects could arise in the process of collapse of massive fast-rotating stars or in the mergers of neutron stars, which was directly observed on August 17, 2017 (Abbot et al., 2017 [69]). Here we only pay attention to several works devoted to the study of the collapse of such objects from the point of view of applying the results obtained to gamma-ray bursts (see Lipunov, 1987 [15]; Lipunova, 1997 [70]; Lipunova, Lipunov in 1998 [71]; Lipunov,Gorbovskoy in 2007 [72] and in 2008 [73]; Lipunova 2009 [74]).

The future development of the Gravity Magnetic Rotator model can be viewed from two different points of view. First, it is a more adequate and detailed description of the individual modes of interaction of GMR with the surrounding plasma. In fact, this will give a good result only for some classes of objects, for example, if we examine in detail a particular stage in the evolution of a binary system. For example, cataclysmic 
variables, their mass distributions, orbit periods, etc. However, it should be borne in mind here that various uncertainties of the modern evolutionary scenario (such as an adequate description of the common shell stage, the Roche lobe overflow process, magnetic stellar wind parameters and t. d.) could hardly have made the detailed calculations substantially more accurate than they are already (i.e. they remain undefined with the same coefficient 2). On the other hand, we are counting on progress in the study of extragalactic binary systems, with a gradual transition to the evolution of the baryon component of the Universe.

The work was supported by RFBR grant 17-52-80133

References

[1] N.S.Kardashev "Magnetic Collapse and the Nature of Intense Sources of Cosmic Radio-Frequency Emission" , AZh, 41, 807K [1964]

[2] F. Pacini. "Energy Emission from a Neutron Star" Nature, 216, 567 (1967)

[3] Hewish, A.; Bell, S. J.; Pilkington, J. D. H.; Scott, P. F.; Collins, R. A. "Observation of a Rapidly Pulsating Radio Source" Natur, 217, 709H [1968]

[4] T. Gold, "Rotating Neutron Stars as the Origin of the Pulsating Radio Sources" Nature, 218, 731G (1968)

[5] Ya.B. Zel'dovich, I.D.Novikov, “ $\quad$ Reviews of Topical Problems: Relativistic Astrophysics” Soviet Physics Uspekhi, V. 8 (4), 522-577 (1966).

[6] Shklovsky I. "On the Nature of the Source of X-Ray Emission of Sco XR-1." 1967ApJ...148L...1S

[7] Ya.B.Zeldovich "The Fate of a Star and the Evolution of Gravitational Energy Upon Accretion" SPhD, 9, 195Z (1964)

[8] E.E.Salpeter "Accretion of interstellar matter by massive objects" ApJ, 140, 796 (1964)

[9] R.Giacconi, S.Murray, H.Gursky, E.Kellogg, E.Schreier, H.Tananbaum, “The Uhuru catalog of X-ray sources.” ApJ, 178, 281G (1972)

[10] P.R.Amnuel, Guseinov O., H., "X-ray emission during accretion of the interstellar matter by neutron star". Notices (Izvestia) of Azerbadzhan Academy of Science, Physics and Mathematic series, 1968, N.3, 70-74 (1968)

[11] G.S.Bisnovatiy-Kogan, A.M.Friedman, "A Mechanism for Emission of X Rays by a Neutron Star" AZh, 46, 721 (1969)

[12] Ya.B.Zeldovich, N.I.Shakura, "X-Ray Emission Accompanying the Accretion of Gas by a Neutron Star" SvA, 13, 175Z (1969) 
[13] V. Shvartsman, "Two generations of pulsars" Radiophysics and Quantum Electronics, V.13 (12), 1428-1440 (1970)

[14] V.M.Lipunov "The universal diagram for magnetized neutron stars in the galaxy" Ap\&SS, 85, 451L (1982)

[15] V.M.Lipunov, "The ecology of rotators" Astrophysics and Space Science, 132, 1$50,(1987 a)$

[16] V.M.Lipunov, 1992a, Astrophysics of Neutron Stars, Springer-Verlag, Berlin, Heidelberg, New York. Also Astronomy and Astrophysics Library, 1992, translation of the Astrofizika nejtronnykh zvezd, Moscow, Nauka

[17] N.I.Shakura, R.Syunyaev "Black holes in binary systems. Observational appearance" A\&A, v24, 337 (1973)

[18] V.M.Lipunov, "Supercritical Disk Accretion onto Magnetized Neutron Stars" Soviet Astronomy, v. 26, 54-56 (1982b)

[19] R.E.Davies, J.E.Pringle, "On accretion from an inhomogeneous medium" MNRAS, 191, 599 (1980)s

[20] V.M.Lipunov, K.A.Postnov, M.E.Prokhorov "The Scenario Machine: Binary star population synthesis", Amsterdam: Harwood Academic Publishers (1996) (http://xray.sai.msu.ru/ mystery/articles/review/ )

[21] J.A. Crawford "On the subgiant components of eclipsing binary systems" ApJ, $121,71(1955)$

[22] Snezhko L.I. “ About the evolution of close binary systems" Peremennye zvezdy (Variable stars), v.16, 253-275 (1967)

[23] E.P.J. van den Heuvel "Evolutionary process in X-ray binaries and their progenitor systems” Ann. New York Acad. Sci., v.302, 13-35 (1977)

[24] A.V. Tutukov, L. Yungelson "Evolution of massive close binaries" NInfo, 27, 70T (1973)

[25] A. F. Illarionov, R.Sunyaev, "Why the Number of Galactic X-ray Stars Is so Small? "A\&A, v.39, 185 (1975)

[26] G.S.Bisnovaty-Kogan, B.V.Komberg, "Possible evolution of a binary-system radio pulsar as an old object with a weak magnetic field" SvAL, V.2, 130-132 (1976)

[27] V. G.Kornilov, V.M.Lipunov "Neutron Stars in Massive Binary Systems - Part One - Classification and Evolution" Sov. Astron., 27, 163 (1983a)

[28] V. G.Kornilov, V.M.Lipunov "Neutron Stars in Massive Binary Systems - Part Two - Numerical Modeling” Sov. Astron. 27334 (1983b)

[29] V. G.Kornilov, V.M.Lipunov “ Collapse Anisotropy for Massive Stars” SvA, 28, 402 (1984) 
[30] V. M.Lipunov, K. A.Postnov, M. E.Prokhorov, A. I.Bogomazov, "Description of the Scenario Machine" ARep, 53 (10), 915-940 (2009)

[31] S.Johnston, R.N.Manchester, A.G.Lyne et al. "PSR 1259-63 - A binary radio pulsar with a Be star companion" ApJ, 387, L37 (1992)

[32] M.Hakan Erkut, K.Y.Ekşi, M.A.Alpar. "Ultraluminous X-ray sources as supercritical propellers”, eprint arXiv:1708.04502, 2017

[33] J.Casares, I.Negueruela, M.Ribó, I.Ribas, J. M.Paredes, A.Herrero, S.Simón-Díaz "A Be-type star with a black-hole companion" Nature, 505, 378-381 (2014)

[34] M.F.Walker, "Photoelectric Observations of Nova DQ Herculis (1934)" Astrophysical Journal, v. 127, 319 (1958)

[35] V.M.Lipunov, K.A. Postnov, M.E. Prokhorov, I.E. Panchenko, H.E. Jorgensen "Evolution of the Double Neutron Star Merging Rate and the Cosmological Origin of Gamma-Ray Burst Sources” ApJ, 454, 593L (1995)

[36] K. Schenker, A.R. King, et al. "AE Aquarii: how cataclysmic variables descend from supersoft binaries" MNRAS 337, 1105 (2002)

[37] V.M.Lipunov, K.A.Postnov, "The joint evolution of normal and compact magnetized stars in close binaries - Analytical description and statistical simulation" Astrophysics and Space Science, v.145, 1-45 (1988)

[38] V.M.Lipunov, S.N.Nazin ""Recycled" white dwarfs?" Astronomical and Astrophysical Transactions, v. 1 (2), 153-157 (1992)

[39] T.R.Marsh et al. "A radio-pulsing white dwarf binary star" Nature, 537, p 374 377 (2016)

[40] V.M.Lipunov et al., "IceCube HESE 160814: MASTER OT J130845.02-323254.9 as the possible source of the high energy neutrino" GCN Circular, 19888, 1 (2016)

[41] D.A.H. Buckley, P.J.Meintjes, S.B.Potter, Marsh, T. R.; Gänsicke, B. T. "Polarimetric evidence of a white dwarf pulsar in the binary system AR Scorpii" Nature Astronomy, V. 1, id. 0029 (2017)

[42] V.F. Suleimanov, V. Doroshenko, K.Werner, "Hard X-ray view on intermediate polars in the Gaia era", MNRAS,482, 3, p 3622-3635 (2019)

[43] V.M.Lipunov, "On the Equilibrium of HERCULES-X-1" Soviet Astronomy, V.31 (2), 167 (1987b)

[44] V.S.Gnusareva, V.M.Lipunov, "Neutron Star Evolution in Eccentric Orbit Binaries" Soviet Astronomy, v.29, 645-649 (1985)

[45] A.Alpar, A.F.Cheng, M.A.Ruderman, J.Shaham, "A new class of radio pulsars" Nature, V. 300, 728-730 (1982) 
[46] D. E.Kolosov, V.M.Lipunov, K.A.Postnov , M. E.Prokhorov, "Recycled radiopulsar reservation in the P-P(dot) diagram" Astronomy and Astrophysics, 215, L21-L23(1989).

[47] N.I.Shakura," The long-period X-ray pulsar 3U 0900-40 - A neutron star with an anomalously strong magnetic field" PAZh, 1, 23S (1975)

[48] V.M.Lipunov, N.I.Shakura, "On the nature of binary-system X-ray pulsars" SvAL, $2,133 \mathrm{~L}(1976)$

[49] V.M.Lipunov, "The magnetic fields of X-ray pulsars" Soviet Astronomy, 26, 537 $541(1982 \mathrm{c})$

[50] Stern, B. E.; Tikhomirova, Ya.; Kompaneets, D.; Stepanov, M.; Berezhnoy, A.; Svensson, R. "Non-Triggered BATSE Gamma-Ray Bursts: An Extended and Revised $\log$ N-log P Distribution" ASPC..190..253S (1999)

[51] E. P. Mazets, S. V. Golenetskii, V. N. Il'inskii, R. L. Aptekar' \& Yu. A. Guryan "Observations of a flaring X-ray pulsar in Dorado" Nature, v.282, 587-589 (1979)

[52] E. P. Mazets, S. V. Golenetskii, V. N. Il'inskii, R. L. Aptekar' \& Yu. A. Guryan "Observations of a flaring X-ray pulsar in Dorado" Nature, v.282, 587-589 (1979)

[53] S.Mereghetti, J.Pons,A. Melatos "Magnetars: Properties, Origin and Evolution" Space Science Reviews, V. 191 (1-4), 315 (2015)

[54] S. A.Olausen; V.M.Kaspi "VizieR Online Data Catalog: The McGill magnetar catalog (Olausen+, 2014)" yCat,22120006O (2014)|

[55] S. A.Olausen; V.M.Kaspi “The McGill Magnetar Catalog” ApJS,212, $6 \mathrm{O}$ (2014)

[56] R.N.Manchester, G. B. Hobbs, et al. "The Australia Telescope National Facility Pulsar Catalogue" AJ, 129, 1993-2006 (2005)

[57] N.V.Raguzova, V.M. Lipunov "High-eccentric X-ray binaries: evolution, wind rose effect, accretor-propeller luminosity gap" A\&A, 340, 85-102 (1998)

[58] A.M. Cherepashchuk et. al, "Highly Evolved Close Binary Stars: Catalog" Advances in astronomy and astrophysics ISBN 90-5699-012-8 (1996)

[59] S.Rappaport, van de Heuvel E. P. J. "X-ray observations of Be stars " in Jaschek M., Groth H. G., eds,IAU Symp. V. 98, Be Stars. Reidel, Dordrecht, p. 327 (1982)

[60] E. P. J.van den Heuvel, S.Rappaport "X-ray observations of B-emission stars " in Slettebak A., Snow T. P. eds. IAU Colloq. V. 92 : Physics of Be Stars. Cambridge Univ. Press, Cambridge, p. 291 (1987)

[61] S. V.Karpov, V.M.Lipunov, "Why Do We See So Few Black Holes in Massive Binaries?" Astronomy Letters, V.27(10), 645-647 (2001) 
[62] R.O.Brown, W. C. G.Ho, M. J.Coe, A. T.Okazaki "Simulating the X-ray luminosity of Be X-ray binaries: the case for black holes versus neutron stars" MNRAS, V. 477 (4),4810-4816 (2018)

[63] G.L. Israel et al. "An accreting pulsar with extreme properties drives an ultraluminous x-ray source in NGC 5907” Sci...355..817I (2017)

[64] G.L. Israel et al. "Discovery of a 0.42-s pulsar in the ultraluminous X-ray source NGC 7793 P13” MNRAS.466L..48I (2017)

[65] M. Brightman et al. "Chandra and NuSTAR studies of the ultraluminous X-ray sources in M82" HEAD...1520703B (2016)

[66] Ya.B.Zeldovich, L.N.Ivanova, D.K.Nadezghin,” Nonstationary Hydrodynamical Accretion onto a Neutron Star.” Astron. Zh., 49, 253-264 (1972)

[67] M.M.Basko , R.A.Sunyaev, "Radiative transfer in a strong magnetic field and accreting X-ray pulsars”Astron. \&Astrophys., 42, 311 (1975)

[68] I.G.Mitrofanov, G.G.Pavlov, Iu. N.Gnedin, "The nature of the AM Herculis system /3U 1809+50" Soviet Astronomy Letters, v. 3, 182-184 (1977)

[69] Abbott et al. "Multi-messenger Observations of a Binary Neutron Star Merger" ApJ, 848, L12 (2017)

[70] G. V. Lipunova, "A burst of electromagnetic radiation from a collapsing magnetized star" Astronomy Letters, V.23(1), 84-92 (1997)

[71] G.V.Lipunova, V.M Lipunov, "Formation of a gravitationally bound object after binary neutron star merging and GRB phenomena" Astronomy and Astrophysics, v.329, p.L29-L32 (1998)

[72] V.M.Lipunov, E.S. Gorbovskoy, "Spinar paradigm and the central engine of gamma-ray bursts" MNRAS, V. 383, 1397 (2008)

[73] V.M.Lipunov, E.Gorbovskoy, “An Extra Long X-Ray Plateau in a Gamma-Ray Burst and the Spinar Paradigm" ApJ, V. 665, L97 (2007)

[74] G.V.Lipunova, E. S.Gorbovskoy, A.I.Bogomazov, V.M.Lipunov , "Population synthesis of gamma-ray bursts with precursor activity and the spinar paradigm" MNRAS, V. 397, 1695 (2009)

[75] Davies, R. E.; Pringle, J. E. MNRAS, 196, 209D (1981) 